\title{
Global representation of Segre numbers by Monge-Ampère products
}

\author{
Mats Andersson ${ }^{1}$. Dennis Eriksson ${ }^{1}$ - Håkan Samuelsson Kalm ${ }^{1}$. \\ Elizabeth Wulcan ${ }^{1}$. Alain Yger $^{2}$
}

Received: 30 January 2019 / Revised: 9 December 2019 / Published online: 10 March 2020

(c) The Author(s) 2020

\begin{abstract}
On a reduced analytic space $X$ we introduce the concept of a generalized cycle, which extends the notion of a formal sum of analytic subspaces to include also a form part. We then consider a suitable equivalence relation and corresponding quotient $\mathcal{B}(X)$ that we think of as an analogue of the Chow group and a refinement of de Rham cohomology. This group allows us to study both global and local intersection theoretic properties. We provide many $\mathcal{B}$-analogues of classical intersection theoretic constructions: For an analytic subspace $V \subset X$ we define a $\mathcal{B}$-Segre class, which is an element of $\mathcal{B}(X)$ with support in $V$. It satisfies a global King formula and, in particular, its multiplicities at each point coincide with the Segre numbers of $V$. When $V$ is cut out by a section of a vector bundle we interpret this class as a Monge-Ampère-type product. For regular embeddings we construct a $\mathcal{B}$-analogue of the Gysin morphism.
\end{abstract}

Communicated by Ngaiming Mok.

Mats Andersson, Håkan Samuelsson Kalm and Elizabeth Wulcan were partially supported by the Swedish Research Council.

\footnotetext{
$凶$ Håkan Samuelsson Kalm

hasam@chalmers.se

Mats Andersson

matsa@chalmers.se

Dennis Eriksson

dener@chalmers.se

Elizabeth Wulcan

wulcan@chalmers.se

Alain Yger

Alain.Yger@math.u-bordeaux.fr

1 Department of Mathematics, Chalmers University of Technology and the University of Gothenburg, S-412 96 Göteborg, Sweden

2 IMB, Université de Bordeaux, 33405 Talence, France
} 


\section{Introduction}

Throughout this paper $X$ is a reduced analytic space of pure dimension $n$ and $\mathcal{J} \rightarrow X$ is a coherent ideal sheaf with zero set $Z$ with codimension $\kappa$. Tworzewski [21], and Gaffney and Gassler [13], independently introduced, at each point $x \in X$, numbers $e_{\kappa}(\mathcal{J}, x), \ldots, e_{n}(\mathcal{J}, x)$ that generalize the Hilbert-Samuel multiplicity at $x$. These definitions, although slightly different, are both of a geometric nature. There is also a purely algebraic definition, see [1,2] by Achilles-Manaresi and Achilles-Rams, respectively. In [5] were introduced semi-global currents whose Lelong numbers at $x$ are precisely the $e_{k}(\mathcal{J}, x)$, thus providing an analytic definition. Following [13] we call these numbers Segre numbers and, indeed, we will see in Theorem 1.1 below that they are closely related to Segre classes.

The main goal in this paper is to define concrete global analytic-geometric objects that represent the Segre numbers at each point. A secondary goal is to provide a framework, based on currents, to connect local intersection theory with global constructions.

Intersection theory deals with the $\mathbb{Z}$-module $\mathcal{Z}(X)$ of analytic cycles and its quotient module $\mathcal{A}(X)$, the Chow group. In general there are no cycles or elements in $\mathcal{A}(X)$ that can represent the Segre numbers at each point. To find global representations we introduce an extension $\mathcal{G} \mathcal{Z}(X)$ of $\mathcal{Z}(X)$ that we call the $\mathbb{Z}$-module of generalized cycles. Formally the elements in $\mathcal{G} \mathcal{Z}(X)$ are a certain kind of closed currents but we prefer to think of them as geometric objects. In particular, ordinary cycles are certainly geometric objects but formally represented by their associated Lelong currents in $\mathcal{G} \mathcal{Z}(X)$. Many of the well-known geometric properties of $\mathcal{Z}(X)$ extend to $\mathcal{G} \mathcal{Z}(X)$ : We have the natural grading by dimension $\mathcal{G} \mathcal{Z}(X)=\oplus_{0}^{n} \mathcal{G Z}_{k}(X)$, where $\mathcal{G Z}_{k}(X)$ are the submodules of generalized cycles of pure dimension $k$. At each point a generalized cycle $\mu$ has a well-defined multiplicity that is an integer. There is a notion of Zariski support of $\mu$, and any $\mu$ has a unique decomposition in irreducible components. Moreover, $\mathcal{G} \mathcal{Z}(X)$ is closed under multiplication by components of Chern and Segre forms of Hermitian vector bundles. ${ }^{1}$ To get independence of various choices we introduce a certain quotient module $\mathcal{B}(X)$ of $\mathcal{G} \mathcal{Z}(X) ; \mathcal{B}(X)$ preserves the above-mentioned geometric properties of $\mathcal{G} \mathcal{Z}(X)$. For instance, $\mathcal{Z}(X)$ is a submodule of $\mathcal{B}(X)$, we have a grading by dimension $\mathcal{B}(X)=\oplus_{0}^{n} \mathcal{B}_{k}(X)$ and well-defined multiplicities, etc. Moreover, $\mathcal{B}(X)$ admits a multiplication by components of Chern and Segre classes. A proper mapping ${ }^{2} f: X^{\prime} \rightarrow X$ induces a mapping $f_{*}: \mathcal{G Z}\left(X^{\prime}\right) \rightarrow \mathcal{G Z}(X)$, which in turn induces a mapping $\mathcal{B}\left(X^{\prime}\right) \rightarrow \mathcal{B}(X)$. Assume that $i: V \hookrightarrow X$ is a subvariety. The image of the injective mapping $i_{*}: \mathcal{G} \mathcal{Z}(V) \rightarrow \mathcal{G} \mathcal{Z}(X)$ is precisely the elements in $\mathcal{G} \mathcal{Z}(X)$ that have Zariski support in $V$. Conceptually we identify $\mathcal{G} \mathcal{Z}(V)$ with its image. In the same way $\mathcal{B}(V)$ is identified with the elements in $\mathcal{B}(X)$ that have Zariski support on $V$.

We define the $\mathcal{B}$-Segre class $S(\mathcal{J}, X)$ in $\mathcal{B}(Z)$ in analogy with the Segre class in $\mathcal{A}(Z)$, cf. Remark 5.1: first assume that $X$ is irreducible. If $\mathcal{J}$ vanishes identically on $X$, then $S(\mathcal{J}, X)=1$ on $X$. Otherwise, let $\pi: X^{\prime} \rightarrow X$ be any modification of $X$

\footnotetext{
1 All vector bundles in this paper are holomorphic.

2 Mappings between spaces are always assumed to be holomorphic.
} 
such that the ideal sheaf $\pi^{*} \mathcal{J}$ is principal, ${ }^{3}$ let $c_{1}(L)$ be the first Chern class of the line bundle $L$ defining the exceptional divisor $D$ in $X^{\prime}$, and let $[D]$ be its Lelong current. For instance, $X^{\prime}$ can be the blow-up of $X$ along $\mathcal{J}$. Then

$$
S(\mathcal{J}, X)=\pi_{*}\left([D] \wedge \frac{1}{1+c_{1}(L)}\right)=\sum_{j=1}^{n}(-1)^{j-1} \pi_{*}\left([D] \wedge c_{1}(L)^{j-1}\right)
$$

Since $\pi$ is proper, (1.1) defines an element in $\mathcal{B}(Z)$. We will see that it is independent of the choice of modification. If $X$ consists of the irreducible components $X_{1}, X_{2}, \ldots$, then we let $S(\mathcal{J}, X)=S\left(\mathcal{J}, X_{1}\right)+S\left(\mathcal{J}, X_{2}\right)+\cdots$ which is a locally finite sum on $X$.

We are now ready to formulate our first main result, which is a generalized King formula, [16,17], for these objects and that in particular provides the desired global representation of the Segre numbers of $\mathcal{J}$. Let $S_{k}(\mathcal{J}, X)$ be the component of $S(\mathcal{J}, X)$ in $\mathcal{B}_{n-k}(Z)$.

Theorem 1.1 (Global generalized King formula) Let $\mathcal{J} \rightarrow X$ be a coherent ideal sheaf over a reduced analytic space of pure dimension $n$ and let $\kappa$ be the codimension of the zero set $Z$ of $\mathcal{J}$. The class $S(\mathcal{J}, X)$ only depends on the integral closure class of $\mathcal{J}$. We have unique decompositions

$$
S_{k}(\mathcal{J}, X)=\sum_{j} \beta_{j}^{k}\left[Z_{j}^{k}\right]+N_{k}^{\mathcal{J}}, \quad k=0,1,2, \ldots,
$$

in $\mathcal{B}_{n-k}(Z)$, where $Z_{j}^{k}$ are the (Fulton-MacPherson) distinguished varieties of $\mathcal{J}$ of codimension $k, \beta_{j}^{k}$ are positive integers, and $N_{k}^{\mathcal{J}}$ has the following property: the multiplicities mult ${ }_{x} N_{k}^{\mathcal{J}}$ are nonnegative integers, and the set of $x$ where mult $_{x} N_{k}^{\mathcal{J}} \geq 1$ has codimension at least $k+1$. Moreover, $S_{k}(\mathcal{J}, X)=0$ for $k<\kappa, N_{\kappa}^{\mathcal{J}}=0$, and

$$
\operatorname{mult}_{x} S_{k}(\mathcal{J}, X)=e_{k}(\mathcal{J}, x), \quad k=\kappa, \ldots, n, \quad x \in X
$$

Our next objective is to present specific representatives for the $\mathcal{B}$-Segre class $S(\mathcal{J}, X)$. Assume that we have a holomorphic section $\sigma$ of a Hermitian vector bundle $E \rightarrow X$ such that $\sigma$ generates $\mathcal{J}$. If $X$ is projective such a $\sigma$ always exists. One can give a meaning to the Monge-Ampère products $\left(d d^{c} \log |\sigma|^{2}\right)^{k}$ for all $k=0,1, \ldots$, as follows. To begin with it is defined as 1 when $k=0$. The higher powers are defined recursively in [3] as

$$
\left(d d^{c} \log |\sigma|^{2}\right)^{k}=d d^{c}\left(\log |\sigma|^{2} \mathbf{1}_{X \backslash Z}\left(d d^{c} \log |\sigma|^{2}\right)^{k}\right) .
$$

\footnotetext{
${ }^{3}$ In this paper we let $\pi^{*} \mathcal{J}$ denote the ideal generated by the pullback of generators of $\mathcal{J}$.
} 
For $k \leq \operatorname{codim} \mathcal{J}$ this definition coincides with Demailly's extension of the classical Bedford-Taylor definition. Proposition 4.4 in [3] states that

$$
\left(d d^{c} \log |\sigma|^{2}\right)^{k}=\lim _{\epsilon \rightarrow 0}\left(d d^{c} \log \left(|\sigma|^{2}+\epsilon\right)\right)^{k},
$$

which gives further motivation for the notation. It was recently proved in [6] that one can also take the limit when $\ell \rightarrow \infty$ of $\left(d d^{c} u_{\ell}\right)^{k}$, where $u_{\ell}=\max \left(\log |\sigma|^{2},-\ell\right)$; several other, but not all (sic!), sequences of plurisubharmonic functions decreasing to $\log |\sigma|^{2}$ also work.

Theorem 1.2 Let $\sigma$ be a holomorphic section of a Hermitian vector bundle $E \rightarrow X$ and let $\mathcal{J}$ be the ideal sheaf generated by $\sigma$. The current

$$
M_{k}^{\sigma}:=\mathbf{1}_{Z}\left(d d^{c} \log |\sigma|^{2}\right)^{k}, \quad k=0,1,2, \ldots,
$$

is a generalized cycle that represents the $\mathcal{B}_{n-k}(Z)$-class $S_{k}(\mathcal{J}, X)$.

Since $\mathcal{Z}(Z)$ is a subgroup of $\mathcal{B}(Z)$ we conclude the following global version of [5, Theorem 1.1] from Theorems 1.1 and 1.2.

Corollary 1.3 We have unique decompositions

$$
M_{k}^{\sigma}=\sum_{j} \beta_{j}^{k}\left[Z_{j}^{k}\right]+N_{k}^{\sigma}, \quad k=\kappa, \ldots, n,
$$

where $N_{k}^{\sigma}$ are elements in $\mathcal{G Z}_{n-k}(Z)$. In particular, mult ${ }_{x} M_{k}^{\sigma}$ is equal to the Segre number $e_{k}(\mathcal{J}, x)$ at each point $x$.

Given a generalized cycle $\mu \in \mathcal{G Z}_{m}(X)$ with Zariski support $|\mu|$ we define in Sect. 5 for each $k \geq 0$ a generalized cycle $M_{k}^{\sigma} \wedge \mu$ with Zariski support on $Z \cap|\mu|$ and dimension $m-k$. Its class in $\mathcal{B}_{m-k}(Z \cap|\mu|)$ only depends on $\mathcal{J}$ and the class of $\mu$ in $\mathcal{B}_{m}(X)$. We let $M^{\sigma} \wedge \mu=M_{0}^{\sigma} \wedge \mu+M_{1}^{\sigma} \wedge \mu+\cdots$. We think of $M^{\sigma} \wedge \mu$ as (the push-forward to $X$ of) a representative of the Segre class $S_{k}(\mathcal{J}, \mu)$ of $\mathcal{J}$ on $\mu$, cf. Remark 5.4.

Notice that a coherent ideal sheaf $\mathcal{J} \rightarrow X$ can be identified with the, possibly nonreduced, embedded space $Z_{\mathcal{J}} \hookrightarrow X$ with underlying reduced space $Z$ and structure sheaf $\mathcal{O}_{X} / \mathcal{J}$. If $i: \mu \hookrightarrow X$ is a reduced analytic subspace, then we denote by $s(\mathcal{J}, \mu)$ the class in $\mathcal{A}(Z)$, called the Segre class, that is denoted by $s\left(Z_{i^{*}} \mathcal{J}, \mu\right)$ in [12], cf. Remark 5.1.

In intersection theory the notion of regular embedding $W \hookrightarrow X$ plays a central role. With the identification above "regular" means that the associated sheaf $\mathcal{J} \rightarrow X$ is locally a complete intersection. ${ }^{4}$ Since our second goal concerns intersection theory we will pay special attention to such sheaves $\mathcal{J}$ and describe $S(\mathcal{J}, X)$ in more detail. In this case the normal cone $N_{\mathcal{J}} X$ is a vector bundle over $Z$ and we let $s\left(N_{\mathcal{J}} X\right)=$ $1+s_{1}\left(N_{\mathcal{J}} X\right)+s_{2}\left(N_{\mathcal{J}} X\right)+\cdots+s_{n-\kappa}\left(N_{\mathcal{J}} X\right)$ be its associated total Segre class.

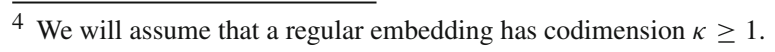


Here lower index $\ell$ denotes the component of bidegree $(\ell, \ell)$. Let $\left[Z_{\mathcal{J}}\right]$ be (the Lelong current of) the fundamental cycle of $Z_{\mathcal{J}}$, cf. [12, Ch. 1.5].

Proposition 1.4 If $\mathcal{J}$ defines a regular embedding $Z_{\mathcal{J}} \hookrightarrow X$, then

$$
S_{k}(\mathcal{J}, X)=s_{k-\kappa}\left(N_{\mathcal{J}} X\right) \wedge\left[Z_{\mathcal{J}}\right], \quad k=\kappa, \ldots, n,
$$

in $\mathcal{B}_{n-k}(X)$.

As in the case with general ideal sheaves we are interested in specific representatives, so let us assume that $\mathcal{J}$ is defined by a section $\varphi$ of a Hermitian vector bundle $F \rightarrow X$ and let $F^{\prime}$ be the pull-back of $F$ to $Z$. There is a canonical holomorphic embedding $i_{\varphi}: N_{\mathcal{J}} X \rightarrow F^{\prime}$ of $N_{\mathcal{J}} X$ in $F^{\prime}$, see Sect. 7 . Let us equip $N_{\mathcal{J}} X$ with the induced Hermitian metric and let $\hat{s}\left(N_{\mathcal{J}} X\right)$ be the associated total Segre form which indeed is smooth on $Z$, see Sect. 2.

Proposition 1.5 If $\varphi$ is a section of the Hermitian vector bundle $F$ defining $\mathcal{J}$, then we have the equality of generalized cycles

$$
M_{k}^{\varphi}=\hat{s}_{k-\kappa}\left(N_{\mathcal{J}} X\right) \wedge\left[Z_{\mathcal{J}}\right], \quad k=\kappa, \ldots, n .
$$

We have a mapping

$$
\mathcal{B}_{k}(X) \rightarrow \mathcal{B}_{k-\kappa}(Z), \quad \mu \mapsto\left(c\left(N_{\mathcal{J}} X\right) \wedge S(\mathcal{J}, \mu)\right)_{k-\kappa},
$$

where lower index denotes dimension, and $c\left(N_{\mathcal{J}} X\right)=1 / s\left(N_{\mathcal{J}} X\right)$ is the total Chern class of $N_{\mathcal{J}} X$. If we choose a section $\varphi$ of $F$ as above we get a representing mapping

$$
\mathcal{G Z}_{k}(X) \rightarrow \mathcal{G Z}_{k-\kappa}(Z), \quad \hat{\mu} \mapsto\left(\hat{c}\left(N_{\mathcal{J}} X\right) \wedge M^{\varphi} \wedge \hat{\mu}\right)_{k-\kappa}
$$

where $\hat{c}\left(N_{\mathcal{J}} X\right)$ is the associated total Chern form. The mapping (1.8) is a $\mathcal{B}$-analogue of the Gysin mapping, [12, Proposition 6.1], see Sect. 2 for the notation,

$$
\mathcal{A}_{k}(X) \rightarrow \mathcal{A}_{k-\kappa}(Z), \quad \mu \mapsto\left(c\left(N_{\mathcal{J}} X\right) \cap s(\mathcal{J}, \mu)\right)_{k-\kappa}
$$

In Sect. 10 we introduce a quotient space $\widehat{H}^{\ell, \ell}(X)$ of closed $(\ell, \ell)$-currents with support on $X$, coinciding with the usual de Rham cohomology in case $X$ is smooth. There are natural mappings $\mathcal{A}_{k}(X) \rightarrow \widehat{H}^{n-k, n-k}(X)$ and $\mathcal{B}_{k}(X) \rightarrow \widehat{H}^{n-k, n-k}(X)$.

Proposition 1.6 For each $k$, the images of $\mathcal{A}_{k}(X)$ and $\mathcal{B}_{k}(X)$ in $\widehat{H}^{n-k, n-k}(X)$ coincide.

Proposition 1.7 Assume that $\mathcal{J} \rightarrow X$ defines a regular embedding $Z_{\mathcal{J}} \hookrightarrow X$ of codimension $\kappa$ and let $Z$ be the (reduced) zero set of $\mathcal{J}$. If $\mu$ is a cycle on $X$, then the images in $\widehat{H}^{*, *}(Z)$ of the Gysin and the $\mathcal{B}$-Gysin mappings, (1.8) and (1.10), respectively, of $\mu$ coincide. 
In Sect. 9 we consider a general ideal sheaf $\mathcal{J} \rightarrow X$ that is generated by a tuple $\sigma=\left(\sigma_{0}, \ldots, \sigma_{m}\right)$ of global sections of a line bundle $L \rightarrow X$. In this situation Stückrad-Vogel [20], introduced an algorithm to produce concrete cycles, StückradVogel cycles, that determine a Chow class $v(\mathcal{J}, L, X)$, which is related to $s(\mathcal{J}, X)$ via van Gastel's formulas [14]. Given a Hermitian metric on $L$ we define a global generalized cycle $M^{L, \sigma}$ by taking a certain mean value of Stückrad-Vogel cycles. If we consider $\sigma$ as a section of $E=\oplus_{0}^{m} L$ we have an analogue of van Gastel's formulas relating $M^{L, \sigma}$ and $M^{\sigma}$ as elements in $\mathcal{G} \mathcal{Z}(X)$.

\section{Preliminaries}

Locally there is an embedding $i: X \rightarrow \Omega \subset \mathbb{C}^{N}$ into an open subset $\Omega \subset \mathbb{C}^{N}$. The sheaf $\mathcal{E}_{X}^{n-\ell, n-k}$ of smooth $(n-\ell, n-k)$-forms on $X$ is by definition the quotient sheaf $\mathcal{E}_{\Omega}^{n-\ell, n-k} / \mathcal{K}$ er $i^{*}$, where $\mathcal{K} e r i^{*}$ is the sheaf of forms $\xi$ on $\Omega$ such that $i^{*} \xi$ vanish on $X_{\text {reg. }}$. Since all embeddings are essentially equivalent, this definition is independent of the choice of embedding. The sheaf $\mathcal{C}_{X}^{\ell, k}$ of currents of bidegree $(\ell, k)$ on $X$ is by definition the dual of $\mathcal{E}_{X}^{n-\ell, n-k}$. Given the embedding $X \rightarrow \Omega$, currents $\mu$ in $\mathcal{C}_{X}^{\ell, k}$ can be identified with currents $\mu^{\prime}=i_{*} \mu$ on $\Omega$ of bidegree $(N-n+\ell, N-n+k)$ that vanish on $\mathcal{K} e r i^{*}$. We say that $\mu$ has order zero if $i_{*} \mu$ has order zero; recall that this means that $i_{*} \mu$ has measure coefficients. A current $\mu$ in $\mathcal{C}_{X}^{n-d, n-d}$ is said to have (complex) dimension $d$. If $f: X \rightarrow X^{\prime}$ is proper, then $f^{*}$ is well-defined on smooth forms and $f_{*}$ is well-defined on currents and preserves dimension, see [4]. If $\mu$ is a current on $X$ and $\eta$ is a smooth form on $X^{\prime}$, then

$$
\eta \wedge f_{*} \mu=f_{*}\left(f^{*} \eta \wedge \mu\right)
$$

Moreover, if $\mu$ has order zero then so has $f_{*} \mu$ and

$$
\mathbf{1}_{V} f_{*} \mu=f_{*}\left(\mathbf{1}_{f^{-1} V} \mu\right)
$$

where $\mathbf{1}_{V}$ is the characteristic function of the analytic subset $V$. If $\mu$ is a closed positive current then so is $f_{*} \mu$. The Lelong number $\ell_{x} \mu$ of $\mu$ at $x$ is defined as the Lelong number of $i_{*} \mu$ at $i(x)$ where $i$ is a local embedding in a smooth manifold, see, e.g., [5, Section 2.2]. If $V$ is a subvariety of $X$ of pure dimension $d \geq 0$, then there is an associated closed positive current of dimension $d$, the Lelong current,

$$
\phi \mapsto[V] \cdot \phi=\int_{V_{\mathrm{reg}}} \phi
$$

Recall that to any Hermitian line bundle $L \rightarrow X$ there is an associated (total) Chern form $\hat{c}(L)=1+\hat{c}_{1}(L)$. If $L^{\prime}$ is the same line bundle but with another Hermitian metric, then there is smooth function $\xi$ on $X$ such that

$$
\hat{c}_{1}\left(L^{\prime}\right)-\hat{c}_{1}(L)=d d^{c} \xi
$$


Assume that $E \rightarrow X$ is a Hermitian vector bundle of rank $r$, and let $\pi: \mathbb{P}(E) \rightarrow X$ be the projectivization of $E$, by which we mean the projective bundle of lines through the origin in $E$. Let $L=\mathcal{O}(-1) \subset \pi^{*} E$ be the tautological line bundle equipped with the induced Hermitian metric, and let $\hat{c}(L)$ be its Chern form. The (total) Segre form of $E$ is defined as

$$
\hat{s}(E)=\pi_{*}(1 / \hat{c}(L)) .
$$

Thus $^{5} \hat{s}(E)=1+\hat{s}_{1}(E)+\hat{s}_{2}(E)+\cdots$ where

$$
\hat{s}_{\ell}(E)=(-1)^{\ell+r-1} \pi_{*} \hat{c}_{1}(L)^{\ell+r-1}
$$

is the component of bidegree $(\ell, \ell)$. It is indeed is a smooth form on $X$ : if $X$ is smooth this follows since $\pi$ is a submersion and in general it follows by embedding $X$ locally in a smooth space and extending $E$ to a Hermitian bundle over this space.

Let $X^{\prime}$ be another analytic space and $f: X^{\prime} \rightarrow X$ a proper mapping. Then the tautological line bundle $L^{\prime} \rightarrow \mathbb{P}\left(f^{*} E\right)$ associated with $\mathbb{P}\left(f^{*} E\right) \rightarrow X^{\prime}$ is the pullback of $L \rightarrow \mathbb{P}(E)$ under the induced map $\tilde{f}: \mathbb{P}\left(f^{*} E\right) \rightarrow \mathbb{P}(E)$ and so $\hat{c}_{1}\left(\tilde{f}^{*} L\right)=$ $\tilde{f}^{*} \hat{c}_{1}(L)$. It follows that

$$
\hat{s}_{k}\left(f^{*} E\right)=f^{*} \hat{s}_{k}(E) .
$$

If $E$ is a line bundle, then $\mathbb{P}(E)=X, L=E$, and hence

$$
\hat{c}(E)=1 / \hat{s}(E) \text {. }
$$

For a general Hermitian vector bundle $E \rightarrow X$ we take (2.7) as the definition of its (total) Chern form. Thus $\hat{c}(E)=1+\hat{c}_{1}(E)+\hat{c}_{2}(E)+\cdots$ where the component $\hat{c}_{k}(E)$ of bidegree $(k, k)$ is a polynomial in the $\hat{s}_{\ell}(E)$. From (2.6) we get

$$
\hat{c}_{k}\left(f^{*} E\right)=f^{*} \hat{c}_{k}(E)
$$

Let $E$ and $E^{\prime}$ be the same bundle but with two different Hermitian metrics and let $L$ and $L^{\prime}$ be the associated Hermitian line bundles over $\mathbb{P}(E)$. In view of (2.3), (2.5) and (2.7) (and that $\pi$ is a submersion) we have, for $k \geq 1$, that

$$
\hat{s}_{k}\left(E^{\prime}\right)-\hat{s}_{k}(E)=d d^{c} \omega_{s}, \quad \hat{c}_{k}\left(E^{\prime}\right)-\hat{c}_{k}(E)=d d^{c} \omega_{c},
$$

for suitable smooth $(k-1, k-1)$-forms $\omega_{s}, \omega_{c}$ on $X$. We let $s_{k}(E)$ and $c_{k}(E)$ denote the cohomology classes, which we for simplicity refer to as the Segre and Chern classes, although we only consider representatives obtained from a Hermitian metric as above.

The Hermitian metric on $E$ determines a Chern connection and thus a curvature tensor $\Theta_{E}$. It is proved in [19, Proposition 6] that the definition used here and the differential-geometric definition of Chern form coincide, that is,

$$
\hat{c}(E)=\operatorname{det}\left(I+(i / 2 \pi) \Theta_{E}\right) .
$$

\footnotetext{
5 It is not obvious that $\hat{s}_{0}(E)=1$; however it follows from the corresponding statement for the Chow class, see [12], or from (2.10).
} 
An analytic $k$-cycle $\mu$ on $X$ is a formal locally ${ }^{6}$ finite linear combination $\sum a_{j} V_{j}$, where $a_{j} \in \mathbb{Z}$ and $V_{j} \subset X$ are irreducible analytic sets of dimension $k$. We let

$$
[\mu]:=\sum a_{j}\left[V_{j}\right]
$$

be its associated Lelong current. Note that if $V_{j}$ has dimension $n$ (the dimension of $X$ ), then $\left[V_{j}\right]=\mathbf{1}_{V_{j}}$. We will denote the $\mathbb{Z}$-module of analytic $k$-cycles on $X$ by $\mathcal{Z}_{k}(X)$. The support $|\mu|$ of the cycle $\mu$ is defined as the union of the $V_{j}$ for which $a_{j} \neq 0$ and it coincides with the support of the current $[\mu]$. Recall that

$$
\operatorname{mult}_{x} \mu=\ell_{x}[\mu]
$$

where $\ell_{x} \gamma$ denotes the Lelong number of the closed positive current $\gamma$ at $x$, and mult $_{x} \mu$ is the multiplicity of $\mu$ at $x$ (defined as in [10, Ch. 2.11.1]), see, e.g., [10, 3.15, Proposition 2].

Let $f: X^{\prime} \rightarrow X$ be a proper mapping. For each irreducible subvariety $V \subset X^{\prime}$, let $\operatorname{deg} f_{V}$ denote the degree of $\left.f\right|_{V}: V \rightarrow f(V)$; if $\operatorname{dim} f(V)<\operatorname{dim} V$ it is defined as zero. The push-forward of $\mu \in \mathcal{Z}_{k}\left(X^{\prime}\right)$ is the cycle

$$
f_{*} \mu=\sum a_{j} \operatorname{deg} f_{V_{j}} f\left(V_{j}\right)
$$

in $\mathcal{Z}_{k}(X)$, see, e.g., [12, Section 1.4]. Since $f_{*}[V]=\operatorname{deg} f_{V}[f(V)]$ it follows that

$$
f_{*}[\mu]=\left[f_{*} \mu\right]
$$

In particular, if $i: X \rightarrow Y$ is an embedding in another reduced space $Y$, then $\mu \in$ $\mathcal{Z}_{k}(X)$ can be regarded as a cycle on $Y$ and $i_{*}[\mu]=[\mu]$. For the rest of this paper we often skip the notation $[\mu]$ and identify a cycle with its Lelong current.

Let $d^{c}=(\partial-\bar{\partial}) / 4 i \pi$ so that ${ }^{7} d d^{c} \log |z|^{2}=[0]$ in $\mathbb{C}$. The Poincaré-Lelong formula, usually stated on a smooth manifold, has an extension to our nonsmooth case (see also Sect. 8). We say that a meromorphic section of a line bundle is non-trivial if it is generically holomorphic and non-vanishing.

Proposition 2.1 (The Poincaré-Lelong formula) Let $h$ be a non-trivial meromorphic section of a Hermitian line bundle $L \rightarrow X$. Then $\log |h|^{2}$ has order zero on $X$,

$$
d d^{c} \log |h|^{2}=\lim _{\epsilon \rightarrow 0} d d^{c} \log \left(|h|^{2}+\epsilon\right)
$$

where $h$ is holomorphic, and there is a cycle divh such that

$$
d d^{c} \log |h|^{2}=[\operatorname{div} h]-\hat{c}_{1}(L) .
$$

In case $X$ is smooth, div $h$ is the usual divisor defined by $h$.

\footnotetext{
6 Algebraic geometry only deals with finite linear combinations, but we use the more "analytic" definition.

7 We write [0] rather than $[\{0\}]$ for the point mass at 0 . 
Proof Let $\pi: X^{\prime} \rightarrow X$ be a smooth modification. Since $\pi^{*} h$ is non-trivial on $X^{\prime}$, $\log \left|\pi^{*} h\right|^{2}$ is locally integrable and hence a current of order 0 . Since $\pi$ is a biholomorphism generically, $\log |h|^{2}=\pi_{*} \log \left|\pi^{*} h\right|^{2}$. Thus $\log |h|^{2}$ has order zero. For the same reason the limit (2.14) holds where $h$ is holomorphic, and $\pi_{*} \hat{c}_{1}\left(\pi^{*} L\right)=\hat{c}_{1}(L)$. By the Poincaré-Lelong formula on a smooth manifold, $d d^{c} \log \left|\pi^{*} h\right|^{2}=\left[\operatorname{div} \pi^{*} h\right]-$ $\hat{c}_{1}\left(\pi^{*} L\right)$. Applying $\pi_{*}$ we get (2.15) with $[\operatorname{div} h]=\pi_{*}\left[\operatorname{div} \pi^{*} h\right]$. It follows from (2.13) that divh is a cycle, and it follows from (2.15) that it is independent of the choice of modification.

Let $i: V \hookrightarrow X$ be a subvariety. If $i^{*} h$ is non-trivial, then we say that divh intersects $V$ properly, and we have the proper intersection $[\operatorname{div} h] \wedge[V]:=i_{*}\left(\operatorname{div} i^{*} h\right)$, cf. [10, Ch 2, 12.3] and Sect. 8. Letting $\log |h|^{2}[V]:=i_{*} \log \left|i^{*} h\right|^{2}$ and noting that $\hat{c}_{1}(L) \wedge[V]=i_{*} \hat{c}_{1}\left(i^{*} L\right)$, we get from (2.15) the formula

$$
d d^{c}\left(\log |h|^{2}[V]\right)=[\operatorname{div} h] \wedge[V]-\hat{c}_{1}(L) \wedge[V] .
$$

Recall that $\mu \in \mathcal{Z}_{k}(X)$ is rationally equivalent to $0, \mu \sim 0$, if there are subvarieties $i_{j}: W_{j} \hookrightarrow X$ of dimension $k+1$ and meromorphic non-trivial functions $g_{j}$ on $W_{j}$, such that, writing $g_{j}$ rather than $i_{j}^{*} g_{j}$ for simplicity,

$$
\mu=\sum_{j}\left(i_{j}\right)_{*}\left[\operatorname{div} g_{j}\right]=\sum_{j}\left(i_{j}\right)_{*} d d^{c} \log \left|g_{j}\right|^{2}=\sum_{j} d d^{c}\left(\log \left|g_{j}\right|^{2}\left[W_{j}\right]\right)
$$

cf. (2.16), where the sums are locally finite. We denote the Chow group of cycles $\mathcal{Z}_{k}(X)$ modulo rational equivalence by $\mathcal{A}_{k}(X)$, cf. [12, Chapter 1.3 ]. Note that if $X$ is irreducible and compact and $\mu$ is a Cartier divisor, then $\mu \sim 0$ precisely if $\mu=[\operatorname{div} g]=d d^{c} \log |g|^{2}$ for some meromorphic function $g$ on $X$, i.e., the line bundle $\mathcal{O}(\mu)$ defined by $\mu$ is trivial. Thus for Cartier divisors (when $X$ is compact), rational equivalence precisely means linear equivalence. If $f: X^{\prime} \rightarrow X$ is a proper mapping and $\mu \sim 0$ in $\mathcal{Z}_{k}\left(X^{\prime}\right)$, then $f_{*} \mu \sim 0$ and thus (2.12) induces a mapping, cf. [12, Theorem 1.4],

$$
f_{*}: \mathcal{A}_{k}\left(X^{\prime}\right) \rightarrow \mathcal{A}_{k}(X) .
$$

Each component $c_{k}(E)$ of a Chern class on $X$ induces a mapping $\mathcal{A}_{*}(X) \rightarrow$ $\mathcal{A}_{*-k}(X), \mu \mapsto c_{k}(E) \cap \mu$, see, [12, Section 3.2]. If $h$ is a nontrivial meromorphic section on $|\mu|$ of a line bundle $L$, then $c_{1}(L) \cap \mu$ is the class in $\mathcal{A}(|\mu|)$ defined by $[\operatorname{div} h] \wedge \mu$.

\section{Generalized cycles}

The generalized cycles is the smallest class of currents that is closed under proper direct images and contains sums of wedge products of Lelong currents and components of Chern forms. More formally, we say that a current $\mu$ in $X$ is a generalized cycle if it is a locally finite linear combination over $\mathbb{Z}$ of currents of the form $\tau_{*} \alpha$, where $\tau: W \rightarrow X$ is a proper map, $W$ is smooth, and $\alpha$ is a product of components of Chern forms for 
various Hermitian vector bundles $E_{j}$ over $W$, i.e.,

$$
\alpha=\hat{c}_{k_{1}}\left(E_{1}\right) \wedge \cdots \wedge \hat{c}_{k_{r}}\left(E_{r}\right)
$$

We will keep this notation throughout this section. Since we can restrict $\tau$ to each connected component of $W$ we can assume that $W$ is connected.

Note that a generalized cycle is a real current of order zero that is closed (in particular it is normal) with components of bidegree $(*, *)$. We let $\mathcal{G Z}_{k}(X)$ denote the $\mathbb{Z}$-module of such currents of (complex) dimension $k$, i.e., of bidegree $(n-k, n-k)$, and let $\mathcal{G Z}(X)=\bigoplus \mathcal{G Z}_{k}(X)$. If $\mu \in \mathcal{G Z}(X)$ and $\gamma$ is a component of a Chern form on $X$, then $\gamma \wedge \mu \in \mathcal{G Z}(X)$. In fact, if $\mu=\tau_{*} \alpha$, where $\tau: W \rightarrow X$, then $\gamma \wedge \mu=\tau_{*}\left(\tau^{*} \gamma \wedge \alpha\right)$, cf. (2.1).

Remark 3.1 In view of (2.7) each form (3.1) is a finite sum of similar forms but with $\hat{c}$ replaced by $\hat{s}$. Morover, we can assume that each factor in (3.1) is the first Chern form of a Hermitian line bundle. To see this it is enough to verify that any $\alpha=\hat{s}_{k_{1}}\left(E_{1}\right) \wedge \cdots \wedge \hat{s}_{k_{t}}\left(E_{t}\right)$, where $E_{j} \rightarrow W$ are Hermitian vector bundles of rank $r_{j}$, is of this form. Let $\pi: W^{\prime} \rightarrow W$ be the fiber product $W^{\prime}=\mathbb{P}\left(E_{1}\right) \times_{W} \cdots \times{ }_{W} \mathbb{P}\left(E_{t}\right)$, let $L_{j}$ be the pullback to $W^{\prime}$ of the tautological bundle $\mathcal{O}(-1) \rightarrow \mathbb{P}\left(E_{j}\right)$, and let $\hat{c}_{1}\left(L_{j}\right)$ be the first Chern form on $L_{j}$ induced by the metric on $E_{j}$. Then, cf. (2.5),

$$
\alpha= \pm \pi_{*}\left(\hat{c}_{1}\left(L_{1}\right)^{k_{1}+r_{1}-1} \wedge \cdots \wedge \hat{c}_{1}\left(L_{t}\right)^{k_{t}+r_{t}-1}\right) .
$$

Lemma 3.2 Let $i: V \hookrightarrow X$ be a subvariety and $\mu \in \mathcal{G} \mathcal{Z}(X)$.

(i) Then $\mathbf{1}_{V} \mu \in \mathcal{G} \mathcal{Z}(X)$.

(ii) If

$$
\mu=\sum_{k}\left(\tau_{k}\right)_{*} \alpha_{k},
$$

where $\tau_{k}: W_{k} \rightarrow X$ are proper, $W_{k}$ are smooth and connected, and $\alpha_{k}$ are as in (3.1), then

$$
\mathbf{1}_{V} \mu=\sum_{\tau_{k}\left(W_{k}\right) \subset V}\left(\tau_{k}\right)_{*} \alpha_{k} .
$$

Proof Since the right hand side of (3.3) is in $\mathcal{G Z}(X)$ by definition, (i) follows from (ii). Assume now that (3.2) holds. By (2.2),

$$
\mathbf{1}_{V} \mu=\sum_{k}\left(\tau_{k}\right)_{*}\left(\mathbf{1}_{\tau_{k}^{-1} V} \alpha_{k}\right)
$$

Assume that $\tau_{k}\left(W_{k}\right) \not \subset V$. Then $\tau_{k}^{-1}(V)$ has positive codimension in $W_{k}$ since $W_{k}$ is connected. Since $\alpha_{k}$ is smooth it follows that $\mathbf{1}_{\tau_{k}^{-1} V} \alpha_{k}=0$, and hence the corresponding term in (3.4), vanishes. Thus (3.3) holds. 
If $i: V \hookrightarrow X$ is a subvariety of $X$, then $[V]=i_{*} \alpha$, where $\alpha=1$, which is the 0 th Chern form of any vector bundle over $V$. Thus we have an embedding

$$
\mathcal{Z}_{k}(X) \rightarrow \mathcal{G Z}_{k}(X)
$$

and we think of $\mathcal{Z}_{k}(X)$ as a subset of $\mathcal{G Z}_{k}(X)$. If $h: X^{\prime} \rightarrow X$ is proper, then we have a natural mapping

$$
h_{*}: \mathcal{G Z}_{k}\left(X^{\prime}\right) \rightarrow \mathcal{G Z}_{k}(X) .
$$

Indeed, if $\mu=\tau_{*} \alpha$, then $h_{*} \mu=(h \circ \tau)_{*} \alpha$ and $h \circ \tau$ is proper. In particular, if $i: V \hookrightarrow X$ is a subvariety of $X$, then we have an injective mapping

$$
i_{*}: \mathcal{G Z}_{k}(V) \rightarrow \mathcal{G} \mathcal{Z}_{k}(X)
$$

Given $\mu \in \mathcal{G} \mathcal{Z}(X)$ there is a smallest variety $|\mu|$, that we call the Zariski support of $\mu$, such that $\mu$ vanishes outside $|\mu|$. In fact, $|\mu|$ is the Zariski closure of the support of $\mu$ as a current.

Example 3.3 Assume that $X$ is irreducible and let $L \rightarrow X$ be the trivial line bundle. Then any smooth function $\varphi$ on $X$ determines a metric $|s|_{L}^{2}=|s|^{2} e^{-\varphi}$ on $L$ with the corresponding first Chern form $d d^{c} \varphi$. Since $\mu:=d d^{c} \varphi$ can vanish on an open subset of $X$ without vanishing identically, it is a non-zero generalized cycle with support strictly smaller than $X$ but with $|\mu|=X$.

Proposition 3.4 (Dimension principle) Assume that $\mu \in \mathcal{G Z}_{k}(X)$ has Zariski support $V$. If $\operatorname{dim} V=k$, then $\mu \in \mathcal{Z}_{k}(X)$. If $\operatorname{dim} V<k$, then $\mu=0$.

Proof Since $\mu$ is closed, of dimension $k$ and order zero it follows from [11, Corollary III.2.14] that it is a sum of various currents $a_{j}\left[V_{j}\right]$ where $V_{j}$ is irreducible of dimension $k$ and $a_{j}$ is a number. By Proposition 6.1 the Lelong number of a generalized cycle is an integer at each point, and it follows that the $a_{j}$ are integers. If $\operatorname{dim} V<k$ it follows from [11, Thm III.2.10] that $\mu=0$.

Example 3.5 If $\mu \in \mathcal{G Z}_{n}(X)$, then $\mu=\sum_{j} a_{j} \mathbf{1}_{X_{j}}$, where $X_{j}$ are the irreducible components of $X$ and $a_{j}$ are integers.

Proposition 3.6 The image of (3.6) is precisely those $\mu \in \mathcal{G Z}_{k}(X)$ such that $|\mu| \subset V$.

Thus we can, and will indeed do, identify generalized cycles on $V$ with generalized cycles in $X$ with Zariski support on $V$.

Proof Assume that $\mu$ is on the form (3.2) and has support on $V$. Since $\mu=\mathbf{1}_{V} \mu$ it follows from Lemma 3.2 that $\mu$ is equal to the right hand side of (3.3). For each of these $\tau_{k}$ we have a factorization $\tau_{k}=i \circ \tau_{k}^{\prime}$ where $\tau_{k}^{\prime}: W_{k} \rightarrow V$ is proper. It follows that

$$
\mu^{\prime}:=\sum_{k}\left(\tau_{k}^{\prime}\right)_{*} \alpha_{k}
$$

is in $\mathcal{G} \mathcal{Z}(V)$ and $\mu=i_{*} \mu^{\prime}$. 
Definition 3.7 We say that $\mu \in \mathcal{G Z}(X)$ is irreducible in $X$ if $|\mu|$ is irreducible and $\mathbf{1}_{V} \mu=0$ for any proper subvariety $V \subset|\mu|$.

Thus irreducibility is connected to an irreducible subvariety of $X$. If $\mu \in \mathcal{G} \mathcal{Z}(X)$ is irreducible with Zariski support $V$ it has a unique decomposition

$$
\mu=\mu^{p}+\cdots+\mu^{0}
$$

where $\mu^{k}$ is the component of dimension $k$ and $p=\operatorname{dim} V$. It follows from Proposition 3.4 that $\mu^{p}$ is $a[V]$ for some integer $a$.

Lemma 3.8 Assume that $\mu \in \mathcal{G} \mathcal{Z}(X)$ is of the form $\mu=\tau_{*} \alpha$, where $\tau: W \rightarrow X, W$ is connected, and $\tau(W)=V$. Then $\mu$ is irreducible and $|\mu|=V$ or $\mu=0$.

Proof Since $W$ is irreducible, so is $V$. Clearly, $|\mu| \subset V$. Assume that $V^{\prime}$ is a proper subvariety of $V$. Then $\tau^{-1} V^{\prime}$ has positive codimension in $W$ since $W$ is connected. Thus

$$
\mathbf{1}_{V^{\prime}} \mu=\tau_{*}\left(\mathbf{1}_{\tau^{-1} V^{\prime}} \alpha\right)=0
$$

since $\alpha$ is smooth. If $|\mu|$ is a proper subvariety of $V$, therefore $\mu=\mathbf{1}_{|\mu|} \mu=0$. If not, it follows from (3.8) that $\mu$ is irreducible.

Notice that if $\mu, \mu^{\prime}$ are irreducible with the Zariski support $V$, then $\mu+\mu^{\prime}$ either vanishes or is again irreducible with Zariski support $V$.

Proposition 3.9 Each $\mu \in \mathcal{G} \mathcal{Z}(X)$ has a unique decomposition

$$
\mu=\sum_{j} \mu_{j},
$$

where $\mu_{j} \in \mathcal{G} \mathcal{Z}(X)$ are irreducible with different Zariski supports.

Proof We first prove the uniqueness. Let $V_{j}=\left|\mu_{j}\right|$. Assume that (3.9) holds with $\mu=0$. If there are non-vanishing $\mu_{j}$ then we can choose $k$ such that $\mu_{k} \neq 0$ and $V_{k}$ has minimal dimension among the $V_{j}$ for which $\mu_{j} \neq 0$. For each $j \neq k$ then $V_{k} \cap V_{j}$ has positive codimension in $V_{j}$ and hence $\mathbf{1}_{V_{k}} \mu_{j}=\mathbf{1}_{V_{k} \cap V_{j}} \mu_{j}=0$ since $\mu_{j}$ is irreducible. Thus $\mu_{k}=\mathbf{1}_{V_{k}} \mu_{k}=\mathbf{1}_{V_{k}} \mu=0$ which is a contradiction. We conclude that $\mu_{j}=0$ for all $j$.

To prove the existence, we may assume that $\mu$ is of the form (3.2), where $\tau_{k}$ : $W_{k} \rightarrow X$ and $W_{k}$ are connected. For each subvariety $V_{j} \subset X$ that appears as the Zariski support of one of the summands in (3.2), let $\mu_{j}=\sum\left(\tau_{k}\right)_{*} \alpha_{k}$, where the sum is over all $k$ such that $\tau_{k}\left(W_{k}\right)=V_{j}$. Then, by Lemma 3.8, $\mu_{j}$ is irreducible with Zariski support $V_{j}$ or $\mu_{j}=0$. We now get the decomposition (3.9).

Remark 3.10 It follows from the proof that an irreducible $\mu \in \mathcal{G} \mathcal{Z}(X)$ with $|\mu|=V$ is a finite sum of terms like $i_{*} \tau_{*} \alpha$ where $\tau: W \rightarrow V$ is proper, $\tau(W)=V$ and $W$ is irreducible. Since $\tau$ is proper it is a submersion outside an analytic set $\tau^{-1} V^{\prime}$, where $V^{\prime} \subset V$ has positive codimension, so that $\gamma=\tau_{*} \alpha$ is closed and smooth on $V \backslash V^{\prime} . \square$ 
Given $\mu \in \mathcal{G} \mathcal{Z}(X)$, for each each of the irreducible components $\mu_{j}$ in (3.9) consider the decomposition $\mu_{j}^{p_{j}}+\cdots+\mu_{j}^{0}$ as in (3.7). We have the unique decomposition

$$
\mu=\mu_{f i x}+\mu_{m o v}
$$

where

$$
\begin{gathered}
\mu_{f i x}:=\sum_{j} \mu_{j}^{p_{j}}, \\
\mu_{m o v}=\sum_{j} \sum_{k<p_{j}} \mu_{j}^{k},
\end{gathered}
$$

are called the fixed and moving part of $\mu$, respectively. Notice that $\mu_{f i x}$ is a cycle in view of the dimension principle. We say that each term in (3.11) is a fixed component and each term in (3.12) a moving component of $\mu$. The reason for this terminology will be clarified in Sect. 9 but already here we can present an illustrating example of a moving generalized cycle.

Example 3.11 Assume that $X=\mathbb{P}_{\left[z_{0}: \ldots . z_{n}\right]}^{n}$ and let $\theta=d d^{c} \log \left(\left|z_{1}\right|^{2}+\cdots+\left|z_{n}\right|^{2}\right)$. Then $\theta^{n-k}, k \geq 1$, is a generalized cycle in $\mathbb{P}^{n}$ of dimension $k$ and with Zariski support $\mathbb{P}^{n}$. To see this, let $\pi: B l_{p} \mathbb{P}^{n} \rightarrow \mathbb{P}^{n}$ be the blow-up at $p=[1: 0: \cdots: 0]$ and notice that $\theta=\pi_{*} \hat{\omega}$, where $\hat{\omega}$ is minus the first Chern form of the line bundle, with respect to the "standard" metric, associated with the exceptional divisor $D$. By repeated use of (2.1) we have that $\theta^{n-k}=\pi_{*} \hat{\omega}^{n-k}$ outside the origin. Since both sides are positive closed currents it follows by the dimension principle that the equality must hold across $p$. Thus $\theta^{n-k}$ is in $\mathcal{G} \mathcal{Z}_{k}\left(\mathbb{P}^{n}\right)$ and by Lemma 3.8 it is irreducible with Zariski support $\mathbb{P}^{n}$. Thus it has one single moving irreducible component. One can verify that $\theta^{n-k}$ is indeed a mean value of all $k$-planes through $p$, cf. [5, Eq. (6.2)] with $f=$ $\left(z_{1}, \ldots, z_{n}\right)$. More conceptually, one can thus think of $\theta^{n-k}$ as such a $k$-plane moving around $p$.

\section{Equivalence classes of generalized cycles}

If $0 \rightarrow S \rightarrow E \rightarrow Q \rightarrow 0$ is a short exact sequence of Hermitian vector bundles over $X$ we say that

$$
\hat{c}(E)-\hat{c}(S) \wedge \hat{c}(Q)
$$

is a $B$-form on $X$. Let $\beta$ be the component of bidegree $(k, k)$ of a $B$-form. If $k=0$ then $\beta=0$ so let us assume that $k \geq 1$. In view of (2.10) one can just as well use the differential-geometric definition of Chern form. From [9, Proposition 4.2] we get a smooth form $\gamma$ on $X$ of bidegree $(k-1, k-1)$ such that $\beta=d d^{c} \gamma$. In fact in [9] only the case when $X$ is smooth is discussed. However, the construction of $\gamma$ is completely explicit and local, and locally we can extend our short exact sequence to a neighborhood in a smooth ambient space and conclude that $\gamma$ is smooth on $X$.

Notice for future reference that if $\tau: W \rightarrow X$, then $\tau^{*} \beta$ is a component of a $B$-form if $\beta$ is. We say that $\mu \in \mathcal{G} \mathcal{Z}_{k}(X)$ is equivalent to 0 in $X, \mu \sim 0$, if $\mu$ is a locally finite 
sum of currents of the form

$$
\rho=\tau_{*}(\beta \wedge \alpha)=d d^{c} \tau_{*}(\gamma \wedge \alpha),
$$

where $\tau: W \rightarrow X$ is proper, $W$ is smooth and connected, $\beta$ is a component of a $B$ form on $W$, and $\alpha$ is a product of components of Chern forms. If $\mu=\mu_{0}+\mu_{1}+\cdots$, where $\mu_{k} \in \mathcal{G Z}_{k}(X)$, we say that $\mu \sim 0$ if $\mu_{k} \sim 0$ for each $k$. Let $\mathcal{B}(X)$ denote the $\mathbb{Z}$-module of generalized cycles on $X$ modulo this equivalence. A class $\mu \in \mathcal{B}(X)$ has pure dimension $k, \mu \in \mathcal{B}_{k}(X)$, if $\mu$ has a representative in $\mathcal{G Z}_{k}(X)$. Thus $\mathcal{B}(X)=$ $\oplus_{k} \mathcal{B}_{k}(X)$.

If $E \rightarrow X$ is a Hermitian vector bundle, then for each $k$ we have the mapping

$$
\hat{c}_{k}(E) \wedge: \mathcal{G} \mathcal{Z}_{*}(X) \rightarrow \mathcal{G} \mathcal{Z}_{*-k}(X), \quad \mu \mapsto \hat{c}_{k}(E) \wedge \mu
$$

Proposition 4.1 The mapping (4.3) induces a mapping

$$
c_{k}(E) \wedge: \mathcal{B}_{*}(X) \rightarrow \mathcal{B}_{*-k}(X)
$$

with the following properties: If $F \rightarrow X$ is another vector bundle, then

$$
c_{\ell}(F) \wedge c_{k}(E) \wedge \mu=c_{k}(E) \wedge c_{\ell}(F) \wedge \mu
$$

If $f: W \rightarrow X$ is proper, then

$$
f_{*}\left(c_{k}\left(f^{*} E\right) \wedge \mu\right)=c_{k}(E) \wedge f_{*} \mu
$$

for $\mu \in \mathcal{B}(W)$. If $0 \rightarrow S \rightarrow E \rightarrow Q \rightarrow 0$ is a short exact sequence on $X$, then

$$
c_{k}(E) \wedge \mu=(c(S) \wedge c(Q))_{k} \wedge \mu
$$

Proof First assume that $\hat{\mu} \in \mathcal{G} \mathcal{Z}(X)$ and $\hat{\mu} \sim 0$. With the notation above we may assume that $\hat{\mu}=\tau_{*}(\beta \wedge \alpha)$, where $\tau: W \rightarrow X$ and $\tau$ is a $B$-form on $W$. It follows that $\hat{c}_{k}(E) \wedge \hat{\mu}=\tau_{*}\left(\beta \wedge \hat{c}_{k}\left(\tau^{*} E\right) \wedge \alpha\right)$ and hence by definition $\sim 0$. Thus $\hat{c}_{k}(E) \wedge$ is well-defined on $\mathcal{B}(X)$. We must verify that it does not depend on the particular choice of metric on $E$. To this end, assume that $0 \rightarrow S \rightarrow E \rightarrow Q \rightarrow 0$ is a short exact sequence of Hermitian vector bundles on $X$ and let $\beta$ be the component of bidegree $(k, k)$ of the associated $B$-form. Assume that $\tau: W \rightarrow X$ and $\hat{\mu}=\tau_{*} \alpha$ is an element in $\mathcal{G} \mathcal{Z}(X)$. Then $0 \rightarrow \tau^{*} S \rightarrow \tau^{*} E \rightarrow \tau^{*} Q \rightarrow 0$ is a short exact sequence on $W$ and $\tau^{*} \beta$ is the component of bidegree $(k, k)$ of the associated $B$-form on $W$. It follows that

$$
\beta \wedge \hat{\mu}=\tau_{*}\left(\tau^{*} \beta \wedge \alpha\right) \sim 0 .
$$

If $S=0$ so that $E$ and $Q$ are isomorphic but with possibly different metrics, then $\beta=\hat{c}_{k}(E)-\hat{c}_{k}(Q)$ so we can conclude that $\hat{c}_{k}(E) \wedge \hat{\mu}-\hat{c}_{k}(Q) \wedge \hat{\mu}=0$ in $\mathcal{B}(X)$. Thus (4.4) is well-defined. Now (4.5) and (4.6) are obvious and (4.7) follows from (4.8). $\square$ 
Remark 4.2 If $\beta$ is a component of (4.1), but where all $\hat{c}$ are replaced by $\hat{s}$, then still $\beta \wedge \alpha \sim 0$. In fact, if lower index $\ell$ denotes component of bidegree $(\ell, \ell)$, then

$$
(\hat{s}(E)-\hat{s}(S) \wedge \hat{s}(Q))_{k}=\sum_{\ell=0}^{k}(\hat{c}(E)-\hat{c}(S) \wedge \hat{c}(Q))_{\ell} \wedge(\hat{s}(E) \wedge \hat{s}(S) \wedge \hat{s}(Q))_{k-\ell}
$$

so the claim follows from Remark 3.1. It is clear that Proposition 4.1 holds, with the same proof, if $c$ is replaced by $s$.

Notice that if $h: X^{\prime} \rightarrow X$ is a proper mapping and $\mu \sim 0$, then $h_{*} \mu \sim 0$ so we have a natural mapping $h_{*}: \mathcal{B}(X) \rightarrow \mathcal{B}\left(X^{\prime}\right)$.

Lemma 4.3 If $i: V \hookrightarrow X$ is a subvariety, then $i_{*}: \mathcal{B}(V) \rightarrow \mathcal{B}(X)$ is injective.

Proof Assume that $\mu \in \mathcal{G} \mathcal{Z}(V)$ and $i_{*} \mu \sim 0$ in $\mathcal{G} \mathcal{Z}(X)$. Then $i_{*} \mu=\sum \rho_{j}$, where $\rho_{j}=\left(\tau_{j}\right)_{*}\left(\beta_{j} \wedge \alpha_{j}\right), \tau_{j}: W_{j} \rightarrow X$, are as in (4.2). In view of Lemma 3.2 we may assume that $\tau_{j}\left(W_{j}\right) \subset V$ for each $j$. For each $j$ there is a map $\tau_{j}^{\prime}: W_{j} \rightarrow V$ such that $\tau_{j}=i \circ \tau_{j}^{\prime}$. Let $\rho_{j}^{\prime}=\left(\tau_{j}^{\prime}\right)_{*}\left(\beta_{j} \wedge \alpha_{j}\right)$. Then

$$
i_{*} \mu=\sum_{j} \rho_{j}=i_{*} \sum_{j} \rho_{j}^{\prime},
$$

so that $\mu=\sum_{j} \rho_{j}^{\prime}$. Thus $\mu \sim 0$ on $V$.

Proposition 4.4 The mapping $\mathcal{Z}(X) \rightarrow \mathcal{B}(X)$ is injective.

Thus we can consider $\mathcal{Z}(X)$ as a subgroup of $\mathcal{B}(X)$.

Proof Assume that $\mu=\sum_{j} a_{j} W_{j} \in \mathcal{Z}_{k}(X)$ and $\mu \sim 0$ in $\mathcal{G Z}_{k}(X)$. If $i:|\mu| \rightarrow X$ is the natural injection and

$$
\mu^{\prime}=\sum a_{j} \mathbf{1}_{W_{j}}
$$

then $\mu=i_{*} \mu^{\prime}$. By Lemma 4.3, $\mu^{\prime} \sim 0$ in $\mathcal{G} \mathcal{Z}(|\mu|)$. Since $\hat{\mu}$ has full dimension in $|\mu|$, and thus bidegree $(0,0)$, it must vanish in view of (4.2).

Proposition 4.5 For each open subset $U$ of $X$ there is a natural restriction mapping $r: \mathcal{G Z}(X) \rightarrow \mathcal{G Z}(U)$ that induces a mapping $r: \mathcal{B}(X) \rightarrow \mathcal{B}(U)$.

Proof Assume that $\mu \in \mathcal{G} \mathcal{Z}(X)$ and $\mu=\tau_{*} \alpha$. Then the restriction of the current $\mu$ to $U$ is equal to $\tau_{*}^{\prime} \alpha^{\prime}$, where $\tau^{\prime}$ and $\alpha^{\prime}$ are the restrictions to $U^{\prime}:=\tau^{-1} U$ of $\tau$ and $\alpha$, respectively. Notice that $\tau^{\prime}: U^{\prime} \rightarrow U$ is proper and that $\alpha^{\prime}$ is a product of components of Chern forms since $\alpha$ is. Since also the restriction to $U^{\prime}$ of a $B$-form is a $B$-form, it follows that $r$ is well-defined on $\mathcal{B}(X)$.

Lemma 4.6 Assume that $\mu \in \mathcal{G} \mathcal{Z}(X), \mu \sim 0$, and that (3.9) is its decomposition in irreducible components. Then $\mu_{j} \sim 0$ for each $j$. 
Proof Using the notation from above, we can assume that $\mu$ is of the form

$$
\mu=\sum_{\ell}\left(\tau_{\ell}\right)_{*}\left(\beta_{\ell} \wedge \alpha_{\ell}\right),
$$

where $\tau_{\ell}: W_{\ell} \rightarrow X$ are proper and $W_{\ell}$ are connected. It follows from the proof of Proposition 3.9 that

$$
\mu_{j}=\sum_{\tau_{\ell}\left(W_{\ell}\right)=\left|\mu_{j}\right|}\left(\tau_{\ell}\right)_{*}\left(\beta_{\ell} \wedge \alpha_{\ell}\right)
$$

and thus $\mu_{j} \sim 0$ by definition.

Let $\hat{\mu}$ be a representative of $\mu \in \mathcal{B}(X)$ and let $\hat{\mu}=\sum_{j} \hat{\mu}_{j}$ be its decomposition in irreducible components. We claim that for each $j$ the corresponding class $\mu_{j}$ in $\mathcal{B}(X)$ is independent of the choice of $\hat{\mu}$. In fact, assume that $\hat{v}$ is another representative with decomposition $\sum_{\ell} \hat{v}_{\ell}$. The sums are (locally) finite and each term corresponds to a unique irreducible set, so by adding terms 0 if necessary we have that

$$
\sum_{j}\left(\hat{\mu}_{j}-\hat{v}_{j}\right) \sim 0
$$

and hence by the lemma $\hat{\mu}_{j}-\hat{v}_{j} \sim 0$ for each $j$. Now the claim follows, and taking into account only the nonvanishing classes we get the unique decomposition

$$
\mu=\sum_{j} \mu_{j},
$$

where $\mu_{j}$ are well-defined elements in $\mathcal{B}(X)$ with well-defined Zariski supports $\left|\mu_{j}\right|$.

In case this sum consists of just one non-zero term we thus have a well-defined irreducible subvariety, and so the following definition is meaningful:

Definition 4.7 We say that $\mu \in \mathcal{B}(X)$ is irreducible if it has a representative $\hat{\mu} \in$ $\mathcal{G Z}(X)$ that is irreducible. The Zariski support $|\mu|$ of $\mu$ is then equal to $|\hat{\mu}|$.

We have the following simple consequences of the discussion above:

Proposition 4.8 (i) If $\mu \in \mathcal{B}(X)$ is irreducible and $p=\operatorname{dim}|\mu|$, then we have a unique decomposition $\mu=\mu^{p}+\cdots+\mu^{0}$, where $\mu^{k} \in \mathcal{B}_{k}(X)$.

(ii) Any $\mu \in \mathcal{B}(X)$ has a unique decomposition $\mu=\mu_{1}+\mu_{2}+\cdots$, where $\mu_{j} \in \mathcal{B}(X)$ are irreducible.

Definition 4.9 In view of (ii) we define the Zariski support $|\mu|$ as the union of the $\left|\mu_{j}\right|$.

From Proposition 3.6 and Lemma 4.3 we get

Proposition 4.10 If $i: V \hookrightarrow X$, then the image of $i_{*}: \mathcal{B}(V) \rightarrow \mathcal{B}(X)$ is precisely the $\mu$ in $\mathcal{B}(X)$ with Zariski support on $V$. 
That is, we can identify the elements in $\mathcal{B}(V)$ with elements in $\mathcal{B}(X)$ with Zariski support contained in $V$.

Precisely as for generalized cycles we define $\mu_{\text {fix }}$ and $\mu_{m o v}$ by (3.11) and (3.12), respectively, and get the unique decomposion, cf. (3.10),

$$
\mu=\mu_{f i x}+\mu_{m o v}
$$

in $\mathcal{B}(X)$ in a fixed and a moving part, and in view of Proposition 4.4 the fixed part is indeed a cycle in $X$.

Remark 4.11 Let $X$ be compact, $L \rightarrow X$ be a line bundle, and $\omega=c_{1}(L)$. The mass

$$
a:=\int_{X} \mu \wedge \omega^{j}
$$

of $\mu \in \mathcal{G Z}_{j}(X)$ is an integer that only depends on the class of $\mu$ in $\mathcal{B}_{j}(X)$ and of $L$. In fact, we may assume that $\mu=\tau_{*} \alpha$, where $\alpha$ is a product of first Chern forms of line bundles over $W$ and $\tau: W \rightarrow X$ is proper. Then $\mu \wedge \omega^{j}=\tau_{*}\left(\alpha \wedge \tau^{*} \omega^{j}\right)$ and thus

$$
a=\int_{W} \alpha \wedge \tau^{*} \hat{\omega}^{j}
$$

which is an integer since it is the integral of a product of first Chern forms of line bundles and thus an intersection number. By (4.2) and Stokes' theorem it only depends on the class of $\mu$ and of $L$. When $j=0$ and $\operatorname{dim}|\mu|>0$ we think of $\mu$ as $a$ points moving around on $|\mu|$, cf. Sect. 9 .

\section{The $\mathcal{B}$-Segre class}

Since any modification $\pi: X^{\prime} \rightarrow X$ such that $\pi^{*} \mathcal{J}$ is principal factorizes over the blow-up $B l_{\mathcal{J}} X$ of $X$ along $\mathcal{J}$, it follows by Proposition 4.1 and a standard argument that $S(\mathcal{J}, X)$, as defined in the introduction, cf. (1.1), is a well-defined element in $\mathcal{B}(Z)$. Recall the restriction map $r$ of Proposition 4.5. We claim that

$$
S\left(\left.\mathcal{J}\right|_{U}, U\right)=r S(\mathcal{J}, X)
$$

In fact, by linearity it is enough to check the case when $X$ is irreducible. If $\mathcal{J}$ is the 0 -ideal then (5.1) is trivial. If not, let $\pi: X^{\prime} \rightarrow X$ be a modification such that $\pi^{*} \mathcal{J}$ is principal. Then the restriction $\pi^{\prime}: \pi^{-1} U \rightarrow U$ of $\pi$ is a modification where the pullback of $\left.\mathcal{J}\right|_{U}$ is principal. Let $D^{\prime}$ and $L^{\prime}$ be the restrictions of $D$ and $L$, respectively, to $\pi^{-1} U$. Then

$$
r S(\mathcal{J}, X)=r \pi_{*}\left([D] \wedge \frac{1}{1+c_{1}(L)}\right)=\pi_{*}^{\prime}\left(\left[D^{\prime}\right] \wedge \frac{1}{1+c_{1}\left(L^{\prime}\right)}\right)=S\left(\left.\mathcal{J}\right|_{U}, U\right) .
$$


Remark 5.1 In intersection theory, given a proper subscheme $W \rightarrow X$ there is a welldefined Chow class $s(W, X)$ in $\mathcal{A}(W) \simeq \mathcal{A}(Z), Z=|W|$, called the Segre class. As in the introduction let us think of $W$ as the nonreduced subspace of $X$ with structure sheaf $\mathcal{O}_{W}=\mathcal{O}_{X} / \mathcal{J}_{W}$, where $\mathcal{J}_{W}$ is a coherent ideal sheaf over $X$. Based on Chapter 4 in [12] (the summary on page 70 and Corollary 4.2.2) it follows that $s\left(\mathcal{J}_{W}, X\right):=s(W, X)$ can be defined as $S\left(\mathcal{J}_{W}, X\right)$ in (1.1) if we interpret $c_{1}(L)^{j-1} \wedge[D]$ as the element $c_{1}(L)^{j-1} \cap[D]$ in the Chow group $\mathcal{A}(|D|)$ and $\pi_{*}$ as the push-forward of Chow classes, so that $s_{k}\left(\mathcal{J}_{W}, X\right):=(-1)^{k-1} \pi_{*}\left(c_{1}(L)^{k-1} \cap[D]\right)$ is an element in $\mathcal{A}(Z)$ for $k \geq 1$. Since $W$ is proper, $Z$ has positive codimension and therefore $s_{0}\left(\mathcal{J}_{W}, X\right)$ vanishes.

We shall now discuss concrete representatives of the $\mathcal{B}$-Segre class. In particular, these representations allow us to define the $\mathcal{B}$-Segre class not only on an analytic space but on a generalized cycle $\mu$. To this end we first consider Monge-Ampère products on $\mu$, cf. [5, Sections 5, 6]. Recall that $\sim$ is the equivalence relation defining $\mathcal{B}(X)$.

Theorem 5.2 Assume that $\sigma$ is a holomorphic section of a Hermitian bundle $E \rightarrow X$ and let $\mathcal{J}$ be the associated coherent sheaf with zero set $Z$.

(i) For each $\mu \in \mathcal{G} \mathcal{Z}(X)$ the limits

$$
\left(d d^{c} \log |\sigma|^{2}\right)^{k} \wedge \mu:=\lim _{\epsilon \rightarrow 0}\left(d d^{c} \log \left(|\sigma|^{2}+\epsilon\right)\right)^{k} \wedge \mu, \quad k=0,1,2, \ldots,
$$

exist and are generalized cycles with Zariski support on $|\mu|$, and the generalized cycles

$$
M_{k}^{\sigma} \wedge \mu:=\mathbf{1}_{Z}\left(d d^{c} \log |\sigma|^{2}\right)^{k} \wedge \mu, \quad k=0,1,2, \ldots,
$$

have Zariski support on $Z \cap|\mu|$.

(ii) If $\mu \sim 0$, then $M_{k}^{\sigma} \wedge \mu \sim 0$.

(iii) If $g$ is a holomorphic section of another vector bundle such that ${ }^{8}|\sigma| \sim|g|$, then $M_{k}^{\sigma} \wedge \mu \sim M_{k}^{g} \wedge \mu$.

(iv) If $h: X^{\prime} \rightarrow X$ is proper and $\mu^{\prime} \in \mathcal{G} \mathcal{Z}\left(X^{\prime}\right)$, then

$$
M_{k}^{\sigma} \wedge h_{*} \mu^{\prime}=h_{*}\left(M_{k}^{h^{*} \sigma} \wedge \mu^{\prime}\right)
$$

The hypothesis in (iii), which clearly holds if both $g$ and $\sigma$ define $\mathcal{J}$, precisely means that the sheaves defined by $\sigma$ and $g$ have the same integral closure, see, e.g., [5]. We will refer to (iv) as the projection formula. We let

$$
M^{\sigma} \wedge \mu:=M_{0}^{\sigma} \wedge \mu+M_{1}^{\sigma} \wedge \mu+\cdots
$$

Proof of Theorem 5.2 We can assume that $\mu=\tau_{*} \alpha$, where $\tau: W \rightarrow X$ is proper and $W$ is smooth and connected. We first consider the case when $\tau^{*} \sigma$ vanishes identically on $W$, or equivalently, $|\mu| \subset Z$. For $k \geq 1$ the limit in (5.2) trivially exists and is 0 , and so is (5.3). If $k=0$, then (5.2) is $\mu$ and $M^{\sigma} \wedge \mu=\mathbf{1}_{Z} \mu=\mu$ as well. Thus (i) holds, and (ii)-(iv) are easily verified.

\footnotetext{
${ }^{8}$ Between norms $\sim$ has the standard meaning that there are constants $c, C>0$ such that $c|\sigma| \leq|g| \leq C|\sigma|$.
} 
We can thus assume that $\tau^{*} \sigma$ does not vanish identically on $W$ and hence it defines a subvariety of positive codimension. Then $M_{0}^{\sigma} \wedge \mu=\mathbf{1}_{Z} \mu=0$ since $\mu$ is irreducible, cf. Lemma 3.8. Thus we may assume that $k \geq 1$ and (possibly after a modification of $W$ ) that $\tau^{*} \mathcal{J}$ is principal on $W$. This precisely means that $\tau^{*} \sigma=\sigma^{0} \sigma^{\prime}$, where $\sigma^{0}$ is a section of the line bundle $L_{D} \rightarrow X^{\prime}$ that defines the exceptional divisor $D$ and $\sigma^{\prime}$ is a non-vanishing section of $\tau^{*} E \otimes L_{D}^{-1}=\operatorname{Hom}\left(L_{D}, \tau^{*} E\right)$. Thus $\sigma^{\prime}$ defines an isomorphism between $L_{D}$ and a line subbundle of $\tau^{*} E$, and so $L_{D}$ inherits a metric from $\tau^{*} E$ such that $\left|\sigma^{0}\right|=\left|\tau^{*} \sigma\right|$. If we let

$$
\hat{\omega}=-\hat{c}_{1}\left(L_{D}\right)
$$

we have by the Poincaré-Lelong formula that

$$
d d^{c} \log \left|\tau^{*} \sigma\right|^{2}=[D]+\hat{\omega}
$$

By (2.1),

$$
\left(d d^{c} \log \left(|\sigma|^{2}+\epsilon\right)\right)^{k} \wedge \mu=\tau_{*}\left(\left(d d^{c} \log \left(\left|\tau^{*} \sigma\right|^{2}+\epsilon\right)\right)^{k} \wedge \alpha\right) .
$$

By [3, (4.6)],

$\left(d d^{c} \log \left(\left|\tau^{*} \sigma\right|^{2}+\epsilon\right)\right)^{k} \wedge \alpha \rightarrow\left(d d^{c} \log \left|\tau^{*} \sigma\right|\right)^{k} \wedge \alpha=([D]+\hat{\omega}) \wedge \hat{\omega}^{k-1} \wedge \alpha, \quad \epsilon \rightarrow 0$,

where the middle expression is recursively defined by (1.4). The equality is a simple consequence. We conclude that the limit (5.2) exists for each $k \geq 1$ and that

$$
\left(d d^{c} \log |\sigma|^{2}\right)^{k} \wedge \mu=\tau_{*}\left(([D]+\hat{\omega}) \wedge \hat{\omega}^{k-1} \wedge \alpha\right) .
$$

This is a generalized cycles with Zariski support contained in $\tau(W)=|\mu|$, cf. (3.5).

Since $|D|=\tau^{-1} Z$ we have by (2.2) that

$$
M_{k}^{\sigma} \wedge \mu=\mathbf{1}_{Z}\left(d d^{c} \log |\sigma|^{2}\right)^{k} \wedge \mu=\tau_{*}\left([D] \wedge \hat{\omega}^{k-1} \wedge \alpha\right) .
$$

Clearly it is in $\mathcal{G} \mathcal{Z}(X)$ and has Zariski support contained in $Z \cap|\mu|$. Thus (i) is proved.

If $\alpha=\beta \wedge \alpha^{\prime}$ for some component $\beta$ of a $B$-form, then

$$
M_{k}^{\sigma} \wedge \mu=\tau_{*}\left([D] \wedge \hat{\omega}^{k-1} \wedge \beta \wedge \alpha^{\prime}\right),
$$

and hence $\sim 0$. Thus (ii) holds.

If $g$ is a section as in (iii), then we may assume that also $\tau^{*} g=g^{0} g^{\prime}$. Since $|g| \sim|\sigma|$ it follows that $g^{0}$ and $\sigma^{0}$ define the same divisor and hence are sections of the same line bundle. Hence their associated first Chern forms differ by a $B$-form on $W$. In view of (5.9) and (5.4), $M_{k}^{\sigma} \wedge \mu \sim M_{k}^{g} \wedge \mu$ and thus (iii) follows. Finally, we get (iv) from (5.6), with $h$ instead of $\tau$, and (2.1). 
With the notation in the proof we have, cf. (5.9),

$$
M_{k}^{\tau^{*} \sigma} \wedge \alpha=[D] \wedge \hat{\omega}^{k-1} \wedge \alpha .
$$

Moreover, cf. (1.6), by definition

$$
M^{\sigma}=M^{\sigma} \wedge \mathbf{1}_{X}
$$

Proof of Theorem 1.2 We can assume that $X$ is irreducible. If $\sigma$ vanishes identically, then $M_{0}^{\sigma}=1$ and $M_{k}^{\sigma}=0$ for $k \geq 1$, so $M^{\sigma}$ coincides with $S(\mathcal{J}, X)$ in this case. Thus we may assume that $\mathcal{J}$ has positive codimension, and that $\tau: W \rightarrow X$ is a modification such that $\tau^{*} \sigma$ is principal. It then follows from (1.1), (5.4), and (5.9) with $\alpha=1$ that $M_{k}^{\sigma}$ is a representative for $S_{k}(\mathcal{J}, X)$. Thus Theorem 1.2 follows.

Example 5.3 If the proper map $\tau: W \rightarrow X$ is surjective and generically $m$-to- 1 , then $\tau_{*} \mathbf{1}_{W}=m \mathbf{1}_{X}$ and so $m M^{\sigma}=\tau_{*} M^{\tau^{*} \sigma}$.

Remark 5.4 Assume that $i: V \hookrightarrow X$ is a subvariety of pure codimension $p$. By the projection formula, Theorem $5.2(i v)$,

$$
M_{k}^{\sigma} \wedge[V]=i_{*} M_{k}^{i^{*} \sigma}, \quad k=0,1,2, \ldots
$$

Notice that the Segre class $S\left(i^{*} \mathcal{J}, V\right)$ on $V$ for $i^{*} \mathcal{J} \rightarrow V$ is represented by the generalized cycle $M^{i^{*} \sigma}$, cf. Theorem 1.2. With the identification given by Proposition 4.10 of elements in $\mathcal{B}(V)$ with elements in $\mathcal{B}(X)$ with Zariski support on $V$, thus the right hand side of (5.12) is a representative of $S\left(i^{*} \mathcal{J}, V\right)$. Warning: The left hand side of (5.12) is not a product but an operator acting on $[V]$. In general one cannot recover $M^{\sigma}$ from $i_{*} M^{i^{*} \sigma}$, or $S(\mathcal{J}, X)$ from $S\left(i^{*} \mathcal{J}, V\right)$ even if $Z \subset V$. For instance, if $\mathcal{J}$ defines a regular embedding and $Z=V$, then $i_{*} S\left(i^{*} \mathcal{J}, V\right)=[V]$ whereas $S(\mathcal{J}, X)=[V] \wedge s\left(N_{\mathcal{J}} X\right)$, see Proposition 1.4.

In view of (5.12) the following definition is natural.

Definition 5.5 Assume that $\mathcal{J} \rightarrow X$ is defined by the section $\sigma$ of the Hermitian vector bundle $E \rightarrow X$. Given $\mu \in \mathcal{B}_{p}(X)$ and a representative $\hat{\mu} \in \mathcal{G Z}_{p}(X)$, we define the $\mathcal{B}$-Segre class $S_{k}(\mathcal{J}, \mu)$ as the class in $\mathcal{B}_{p-k}(Z \cap|\mu|)$ defined by $M_{k}^{\sigma} \wedge \hat{\mu}$. We let $S(\mathcal{J}, \mu)=S_{0}(\mathcal{J}, \mu)+S_{1}(\mathcal{J}, \mu)+\cdots+S_{p}(\mathcal{J}, \mu)$.

Proposition 5.6 If $\alpha$ is a component of a Chern or Segre form, then

$$
\mathbf{1}_{Z}(\alpha \wedge \mu)=\alpha \wedge \mathbf{1}_{Z} \mu, \quad \mu \in \mathcal{G} \mathcal{Z}(X),
$$

and

$$
M_{k}^{\sigma} \wedge(\alpha \wedge \mu)=\alpha \wedge M_{k}^{\sigma} \wedge \mu, \quad k=0,1,2, \ldots
$$

Proof Assume that $\mu=\tau_{*} a$ and $W$ is connected. Let $\xi=\tau^{*} \alpha$. Now $\mathbf{1}_{\tau^{-1} Z}(\xi \wedge a)=$ $\xi \wedge \mathbf{1}_{\tau^{-1} Z} a$ since both sides vanish if $\tau^{-1} Z$ is a proper subvariety of $W$ and are equal to $\xi \wedge a$ otherwise. Thus (5.13) follows from (2.1) and (2.2), and (5.14) follows from (5.2), (5.3) and (5.13). 
Sometimes it is convenient with a limit procedure that directly gives $M_{k}^{\sigma} \wedge \mu$ without first computing $\left(d d^{c} \log |\sigma|^{2}\right)^{k} \wedge \mu$.

Proposition 5.7 Let $\sigma$ be a holomorphic section of a Hermitian bundle $E \rightarrow X$ and let

$$
M_{k, \epsilon}^{\sigma}=\frac{\epsilon}{\left(|\sigma|^{2}+\epsilon\right)^{k+1}}\left(d d^{c}|\sigma|^{2}\right)^{k}, \quad k=0,1,2, \ldots
$$

If $\mu \in \mathcal{G} \mathcal{Z}(X)$, then for $k \geq 0$,

$$
M_{k}^{\sigma} \wedge \mu=\lim _{\epsilon \rightarrow 0} M_{k, \epsilon}^{\sigma} \wedge \mu, \quad k=0,1,2, \ldots
$$

Using a principalization, the proposition is reduced to the following lemma that can be verified along the same lines as [3, Proposition 4.4], and we omit the details.

Lemma 5.8 Let $s$ be a section of a Hermitian line bundle $L \rightarrow W$ with divs $=D$ and let $\hat{\omega}=-\hat{c}_{1}(L)$. Then

$$
\frac{\epsilon}{\left(|s|^{2}+\epsilon\right)^{k+1}}\left(d d^{c}|s|^{2}\right)^{k} \rightarrow[D] \wedge \hat{\omega}^{k-1}, \quad k \geq 1 .
$$

Remark 5.9 One can define $M^{\sigma} \wedge \mu$ as the value at $\lambda=0$, via analytic continuation from $\operatorname{Re} \lambda \gg 0$, of the expression

$$
M^{\sigma, \lambda} \wedge \mu=\left(1-|\sigma|^{2 \lambda}+\sum_{k \geq 1} \bar{\partial}|\sigma|^{2 \lambda} \wedge \frac{\partial|\sigma|^{2}}{2 \pi i|\sigma|^{2}} \wedge\left(d d^{c} \log |\sigma|^{2}\right)^{k-1}\right) \wedge \mu
$$

see [3, Proposition 4.1] and [5, Section 4].

Remark 5.10 (Comparison to Green forms) Recall that a ( $p-1, p-1)$-current $g$ is a Green current of a closed subvariety $Z$ of codimension $p$ of a complex manifold $X$ if $d d^{c} g+[Z]=\omega$, where $\omega$ is a smooth form. If $g$ is smooth outside $Z$ it is called a Green form. The calculus of Green forms, based on the $*$-product, is an important tool in the study of height in arithmetic intersection theory, see, e.g., [8,15]. In particular, Fulton's intersection theory is recovered in the proper intersection case.

In the case $p=1$, if $s$ is a section of a Hermitian line bundle that defines $Z$, then $g=-\log |s|^{2}$ is a Green form in virtue of the Poincaré-Lelong formula (2.15). In fact, these are the only Green forms in the case $p=1$. The existence of Green forms of so-called logarithmic type for $p>1$ is a more delicate matter, see [8]. That $g$ is of logarithmic type means that $g=\tau_{*} g^{\prime}$ under a proper mapping $\tau: W \rightarrow X$ such that locally in $W$ there are coordinates $z$ such that $g^{\prime}=\sum_{k} a_{k} \log \left|z_{k}\right|^{2}+a_{0}$, where $a_{j}$ are smooth and closed. This can be compared to our definition of generalized cycles.

If $Z$ is defined by the section $\sigma$ of the Hermitian vector bundle $E \rightarrow X$, and $\gamma:=-\log |\sigma|^{2}\left(d d^{c} \log |\sigma|^{2}\right)^{p-1}$, then, cf. (1.4) and Corollary 1.3,

$d d^{c} \gamma=-\left(d d^{c}|\sigma|^{2}\right)^{p}=-\mathbf{1}_{Z}\left(d d^{c}|\sigma|^{2}\right)^{p}-\mathbf{1}_{X \backslash Z}\left(d d^{c}|\sigma|^{2}\right)^{p}=-[Z]-\mathbf{1}_{X \backslash Z}\left(d d^{c}|\sigma|^{2}\right)^{p}$, 
so that $\gamma$ is kind of a Green form. Unless $p=1$, however, $-\mathbf{1}_{X \backslash Z}\left(d d^{c}|\sigma|^{2}\right)^{p}$ is not smooth but only the push-forward under a modification of a smooth form, cf. (5.8).

\section{Multiplicities of a generalized cycle}

In view of (2.11) it is natural to define the multiplicity of $\mu \in \mathcal{G Z}_{k}(X)$ at $x \in X$ as the Lelong number at $x$. However, $\mu$ is not necessarily positive so it is not immediately clear that the Lelong number exists. Here is our formal definition: Let $\sigma_{x}$ be a section of a Hermitian vector bundle in an open neighborhood $U$ of $x$ such that $\sigma_{x}$ generates the maximal ideal $m_{x}$ at $x$. Since $M^{\sigma_{x}} \wedge \mu$ has support at $x$ it follows from the dimension principle, Proposition 3.4, that $M^{\sigma_{x}} \wedge \mu=M_{k}^{\sigma_{x}} \wedge \mu$. Moreover, in view of the proof of this proposition, $M_{k}^{\sigma_{x}} \wedge \mu=a[x]$ for some real number $a$. By Theorem 5.2 (iii), the number $a$ is independent of the choice of $\sigma_{x}$. Part (ii) of the same theorem implies that $a$ only depends on the class of $\mu$ in $\mathcal{B}(U)$. By an argument as in the beginning of Sect. 5 we see that it is also independent of the choice of neighborhood $U$ of $x$. Altogether the definition

$$
\operatorname{mult}_{x} \mu=\int M^{\sigma_{x}} \wedge \mu
$$

is meaningful. If $U$ is small enough we can assume that $E$ is trivial, with a trivial metric, and then mult ${ }_{x} \mu$ coincides with the Lelong number $\ell_{x} \mu$ if $\mu$ is positive, see, e.g., [5, Lemma 2.1] and Remark 5.9.

Proposition 6.1 The multiplicity of $\mu \in \mathcal{G Z}_{k}(X)$ at $x$ is an integer and it only depends on its class in $\mathcal{B}_{k}(X)$.

Proof Let $\mu=\tau_{*} \alpha$, where $\tau: W \rightarrow X$ is proper and $W$ is connected. First assume that $\tau(W)=\{x\}$. Thus $M^{\sigma_{x}} \wedge \mu=\mu$. Since $\tau$ is proper, $W$ is compact, so by (6.1),

$$
\operatorname{mult}_{x} \mu=\int \mu=\int_{W} \alpha
$$

which is an intersection number, cf. Remark 4.11, and hence an integer. Next we assume that $x \in \tau(W)$ and that $\tau(W)$ has positive dimension. As in the proof of Theorem 5.2, with $\sigma=\sigma_{x}$ and $X=U$, cf. (5.9) and (6.1), we can assume that

$$
M^{\sigma_{x}} \wedge \mu=\sum_{k \geq 1} \tau_{*}\left([D] \wedge \hat{\omega}^{k-1} \wedge \alpha\right) .
$$

Only the term with $k=\operatorname{dim} \mu$ can give a contribution and

$$
\operatorname{mult}_{x} \mu=\int M_{k}^{\sigma_{x}} \wedge \mu=\int \tau_{*}\left([D] \wedge \hat{\omega}^{k-1} \wedge \alpha\right) .
$$


Writing $D=a_{1} D_{1}+a_{2} D_{2}+\cdots$, where $D_{j}$ are irreducible and compact, we therefore have that

$$
\operatorname{mult}_{x} \mu=a_{1} \int_{D_{1}} \hat{\omega}^{k-1} \wedge \alpha+\cdots
$$

and hence an integer, since each integral is an intersection number.

Assume that $\mu$ is irreducible. If it has dimension 0 and is moving, i.e., $\operatorname{dim}|\mu|>0$, then mult $x \mu=0$ at each point. In fact, $\left(\right.$ mult $\left._{x} \mu\right)[x]=\mathbf{1}_{x} \mu=0$ by the definition of irreducibility. However, as is illustrated by Example 3.11, if $\mu$ has positive dimension, mult $_{x} \mu$ can be nonzero at certain points even if $\mu$ is moving.

Proof of Theorem 1.1 It is well-known that the blow-up $\pi: B l_{\mathcal{J}} X \rightarrow X$ of $X$ along $\mathcal{J}$ only depends on the integral closure class of $\mathcal{J}$. Since $S(\mathcal{J}, X)$ is defined just in terms of the blow-up, cf. (1.1), it only depends on the integral closure class of $\mathcal{J}$.

By definition the distinguished varieties are precisely the sets $\pi\left(D_{j}\right)$ where $D_{j}$ are the irreducible components of the exceptional divisor $D$ of the blow-up, see, e.g., [18] or [5].

The remaining statements of Theorem 1.1 are purely local and can be verified in the following way: fix a point $x \in X$. In a suitable neighborhood $U$ of $x$ there is a section $\sigma$ of a trivial vector bundle $E \rightarrow U$ that generates $\mathcal{J}$ there. By Proposition 4.5, mult $_{x} S_{k}(\mathcal{J}, X)=$ mult $_{x} S_{k}\left(\left.\mathcal{J}\right|_{U}, U\right)$. If we choose a trivial metric, then $M_{k}^{\sigma}$ coincides with $M_{k}^{\sigma}$ defined in [5], and from [5, Theorem 1.1] we have that mult $M_{k}^{\sigma}=e_{k}(\mathcal{J}, x)$. Because of the uniqueness of the decomposition (3.10) in fixed and moving components applied to $S(\mathcal{J}, X)$ all the statements now follows from [5, Theorem 1.1].

We have the following consequence of Proposition 5.6.

Lemma 6.2 If $\mu \in \mathcal{B}_{k}(X)$ and $\gamma$ is a component of a Chern or Segre form of positive bidegree, then mult $_{x}(\gamma \wedge \mu)=0$ for each $x$.

Proof Let $\sigma_{x}$ generate the maximal ideal at $x$, and write $\gamma=d d^{c} g$ in a neighborhood of $x$. By Proposition 5.6 and Stokes' theorem, noting that $M^{\sigma_{x}} \wedge \mu$ has support at $x$,

$$
\operatorname{mult}_{x}(\gamma \wedge \mu)=\int M^{\sigma_{x}} \wedge(\gamma \wedge \mu)=\int \gamma \wedge M^{\sigma_{x}} \wedge \mu=\int d d^{c}\left(g \wedge M^{\sigma_{x}} \wedge \mu\right)=0 .
$$

Example 6.3 (Example 3.11 continued) It follows from Lemma 6.2 that mult $\theta^{n-k}=$ 0 for $x \neq p$. From the geometric interpretation as a mean value of $k$-planes through $p$, or by a direct computation of $M^{\sigma_{x}} \wedge \theta^{n-k}$, one can verify that mult $p \theta^{n-k}=1$.

In view of Theorem 1.1, mult ${ }_{x}\left(M_{j}^{\sigma} \wedge \mathbf{1}_{X}\right)=$ mult $_{x} M_{j}^{\sigma}=e_{j}(\mathcal{J}, x)$. For a general $\mu$ in $\mathcal{G Z}_{k}(X)$ or $\mathcal{B}_{k}(X)$ we can define the Segre numbers $e_{j}(\mathcal{J}, \mu, x):=\operatorname{mult}_{x}\left(M_{j}^{\sigma} \wedge \mu\right)$. 


\section{Regular embeddings}

Assume that $\mathcal{J} \rightarrow X$ defines a regular embedding $i: Z_{\mathcal{J}} \rightarrow X$ of codimension $\kappa$, cf. the introduction and Remark 5.1. As before $Z$ denotes the associated reduced space, i.e., the zero set of $\mathcal{J}$. It is well-known that $\mathcal{J} / \mathcal{J}^{2}$ is locally free and thus is the sheaf of sections of a vector bundle known as the conormal bundle of $Z_{\mathcal{J}}$ in $X$. We will denote its dual by $N_{\mathcal{J}} X$, refer to it as the normal bundle of $Z_{\mathcal{J}}$ in $X$, and view it as a holomorphic vector bundle over the reduced space $Z$. We will use the following alternative ad hoc definition of $N_{\mathcal{J}} X$ and its sections: a section $\xi$ of $N_{\mathcal{J}} X \rightarrow Z$ is a choice of holomorphic $\kappa$-tuple $\xi(s)$ locally on $Z$ for each local minimal set $s=\left(s_{1}, \ldots, s_{\kappa}\right)$ of generators for $\mathcal{J}$ so that

$$
g \xi(s)=\xi(g s) \text { on } Z
$$

for any locally defined holomorphic matrix $g$ that is invertible in a neighborhood of $Z$. This defines a vector bundle over $Z$ since for any two such choices $s, s^{\prime}$ there is an invertible matrix $g$ such that $s^{\prime}=g s$ on the overlap in a neighborhood of $Z$. The connection between $N_{\mathcal{J}} X$ and $\mathcal{J} / \mathcal{J}^{2}$ is the non-degenerate pairing $\left(\xi(s), h+\mathcal{J}^{2}\right) \mapsto$ $\xi(s) \cdot h_{s}$, where $h_{s}$ is a tuple, unique $\bmod \mathcal{J}^{2}$, such that $h=s \cdot h_{s}$.

Example 7.1 If $Z$ is smooth and $Z_{\mathcal{J}}$ reduced, then for any $s$ as above, the $d s_{j}$ are linearly independent on $Z$ and vanish on $T Z$. Notice that $g d s=d(g s)$ on $Z$. Therefore, $v \mapsto \xi(s):=\left(d s_{1} \cdot v, \ldots, d s_{\kappa} \cdot v\right)$ defines an injective mapping, hence an isomorphism, $\left.T X\right|_{Z} / T Z \rightarrow N_{\mathcal{J}} X$. In this case therefore $N_{\mathcal{J}} X$ is the usual normal bundle of complex differential geometry.

Remark 7.2 Several results of this section are well-known, at least in the algebraic context. For completeness and reference we give analytic proofs.

Lemma 7.3 Assume that $F \rightarrow X$ is a vector bundle with a holomorphic section $\varphi$ that defines $\mathcal{J}$. Then there is a canonical embedding

$$
i_{\varphi}:\left.N_{\mathcal{J}} X \rightarrow F\right|_{Z}
$$

Proof For each minimal set of generators $s$ of $\mathcal{J}$ in some open connected $U \subset X$ there is a unique $\tilde{A}(s)$ in $\operatorname{Hom}\left(U \times \mathbb{C}^{\mathcal{K}},\left.F\right|_{U}\right)$ such that $\tilde{A}(s) s=\varphi$. Set $A(s)=\left.\tilde{A}(s)\right|_{Z} \in$ Hom $\left(Z \times \mathbb{C}^{\kappa},\left.F\right|_{Z}\right)$. Since $\varphi$ generates $\mathcal{J}$ it follows that $A(s)$ is pointwise injective. Since $\varphi=\tilde{A}(g s) g s$ it follows that $A(g s) g=A(s)$. If $\xi$ is a section of $N_{\mathcal{J}} X$ therefore

$$
A(g s) \xi(g s)=A(g s) g \xi(s)=A(s) \xi(s)
$$

Thus we can define $i_{\varphi}$ as

$$
\xi \mapsto i_{\varphi} \xi, \quad \xi(s) \mapsto A(s) \xi(s)
$$

Since $A(s)$ is pointwise injective it follows that $i_{\varphi}$ is injective. 
In particular, if rank $F=\kappa$, then we have an isomorphism

$$
i_{\varphi}:\left.N_{\mathcal{J}} X \simeq F\right|_{Z}
$$

Proof of Proposition 1.5 We let $q: \mathbb{P}(F) \rightarrow X$ be the projectivization of $F$ and let $\mathcal{O}_{F}(-1) \rightarrow \mathbb{P}(F)$ be the tautological line bundle sitting in $q^{*} F$, equipped with the Hermitian metric inherited from $F$. The line bundle $\mathcal{O}_{N_{\mathcal{J}} X}(-1) \rightarrow \mathbb{P}\left(N_{\mathcal{J}} X\right)$ is defined in the same way, with the Hermitian metric inherited from the normal bundle $N_{\mathcal{J}} X \rightarrow Z$, which in turn has the metric induced by (7.2). Moreover, we let $p: B l_{\mathcal{J}} X \rightarrow X$ be the blow-up of $X$ along $\mathcal{J}$ and let $L_{D} \rightarrow B l_{\mathcal{J}} X$ be the line bundle associated with the exceptional divisor. There are injective holomorphic mappings $j, \tilde{j}, \psi, \tilde{\psi}$ such that the diagram

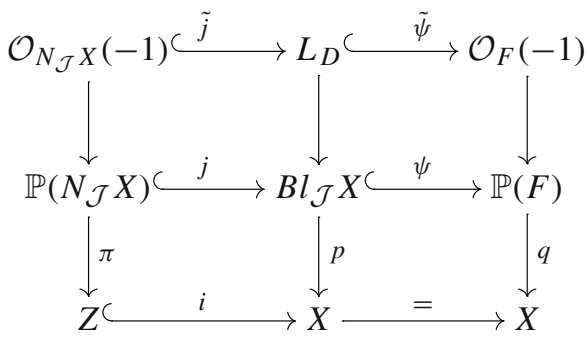

commutes and such that furthermore the Hermitian metric on $\mathcal{O}_{N_{\mathcal{J}} X}(-1)$ coincides with the metric it inherits from $\mathcal{O}_{F}(-1)$ via the first row.

Let us first explain the mapping $j$. Given a minimal set of local generators $s$ of $\mathcal{J}$ as above in say an open set $U \subset X$ we can represent $B l_{\mathcal{J}} U \rightarrow U$ as

$$
B l_{\mathcal{J}} U=\left\{(x,[t]) \in U \times \mathbb{P}^{\kappa-1} ; t_{i} s_{j}(x)-t_{j} s_{i}(x)=0\right\} .
$$

If we choose $s^{\prime}=g s$ in $U^{\prime}$, then we have a similar representation but with $\left[t^{\prime}\right]=[g t]$ on the overlap $U \cap U^{\prime}$. Recall that at $x \in Z$ the fibre of $N_{\mathcal{J}} X$ consists of all $\xi(s) \in \mathbb{C}^{\kappa}$ such that $\xi\left(s^{\prime}\right)=g \xi(s)$, cf. (7.1). We thus have the natural injection

$$
j: \mathbb{P}\left(N_{\mathcal{J}} X\right) \rightarrow B l_{\mathcal{J}} X, \quad(x,[\xi(s)]) \mapsto(x,[t]) .
$$

In $B l_{\mathcal{J}} X \backslash p^{-1} Z$ we define $\psi$ by $\psi\left(p^{-1} x\right)=(x,[\varphi(x)])$. If $x \in U \backslash p^{-1} Z$, then $p^{-1} x=(x,[s(x)])$ and $\tilde{A}(s) s=\varphi$, cf. the proof of Lemma 7.3, so we have that

$$
\psi(x,[t])=(x,[\tilde{A}(s) t])
$$

since $[s(x)]=[t]$. Since $A=\left.\tilde{A}\right|_{Z}$ is injective, (7.8) provides an injective extension of $\psi$ across $p^{-1} Z$ in $U$. This extension is well-defined on overlaps because if $s^{\prime}=g s$, then $\left[t^{\prime}\right]=[g t]$ and by (7.3) hence $\left[A\left(s^{\prime}\right) t^{\prime}\right]=[A(s) t]$. For $x \in Z$ thus $(x,[\xi(s)])$ in $\mathbb{P}\left(N_{\mathcal{J}} X\right)$ is mapped to $(x,[t])$ and by $\psi$ in turn to $(x,[A(s) \xi(s)])$ so the composed mapping $\psi \circ j$ is equal to the mapping $\mathbb{P}\left(N_{\mathcal{J}} X\right) \rightarrow \mathbb{P}(F)$ induced by the canonical embedding $i_{\varphi}$, cf. (7.4). Thus the lower "half" of the diagram is defined and commutes. 
We now define the mapping $\tilde{\psi}$. Since $p^{*} \mathcal{J}$ is principal we recall from the proof of Theorem 5.2 (with $\sigma=\varphi$ and $p^{*} \varphi=\varphi^{0} \varphi^{\prime}$ ) that $L_{D} \rightarrow B l_{\mathcal{J}} X$ can be identified with a line subbundle of $p^{*} E \rightarrow B l_{\mathcal{J}} X$ via the mapping $\varphi^{\prime}$. Since by commutativity $p^{*} F$ is the restriction of $q^{*} F$ to $\psi\left(B l_{\mathcal{J}} X\right)$ we have an injective mapping $L_{D} \hookrightarrow q^{*} F$. We must verify that it actually takes values in $\mathcal{O}_{F}(-1) \subset q^{*} F$. By continuity it is enough to check that this holds over $B l_{\mathcal{J}} X \backslash p^{-1} Z$. However, there the section $\varphi^{0}$ is non-vanishing and mapped onto $\varphi^{0} \varphi^{\prime}=p^{*} \varphi$. Thus $\left(p^{-1} x, \varphi^{0}(x)\right)$ is mapped onto $(x,[\varphi(x)], \varphi(x))$ which is in $\mathcal{O}_{F}(-1)$ by definition.

It remains to explain $\tilde{j}$. Notice that $i_{\varphi}$ induces an embedding $\pi^{*} N_{\mathcal{J}} X \hookrightarrow q^{*} F$ and hence also an embedding $\tilde{i}_{\varphi}: \mathcal{O}_{N_{\mathcal{J}} X}(-1) \hookrightarrow \mathcal{O}_{F}(-1)$. Since $\tilde{\psi}$ is already defined, there is a unique mapping $\tilde{j}$ so that $\tilde{\psi} \circ \tilde{j}=\tilde{i}_{\varphi}$ and the diagram commutes. If $\xi$ is a vector in $\mathcal{O}_{N_{\mathcal{J}} X}(-1)$, then by definition $|\xi|=|\xi|_{N_{\mathcal{J}} X}$ equals $\left|i_{\varphi} \xi\right|_{F}$. However, the norm of $\xi$ induced by the top line is $\left|\tilde{i}_{\varphi} \xi\right|$ which in turn is $\left|i_{\varphi} \xi\right|_{F}$ as well. Thus the claims about (7.6) are proved.

As before, cf. (5.4), we let $-\hat{\omega}=\hat{c}_{1}\left(L_{D}\right)$. By (7.6), $-j^{*} \hat{\omega}$ is the first Chern form of $\mathcal{O}_{N_{\mathcal{J}} X}(-1) \rightarrow \mathbb{P}\left(N_{\mathcal{J}} X\right)$ and so, by definition, cf. (2.4),

$$
\hat{s}\left(N_{\mathcal{J}} X\right)=\pi_{*}\left(\frac{1}{1-j^{*} \hat{\omega}}\right)
$$

Each irreducible component $Z_{\ell}$ of $Z$ corresponds to an irreducible component $D_{\ell}=$ $p^{-1} Z_{\ell}$ of $j\left(\mathbb{P}\left(N_{\mathcal{J}} X\right)\right)=|D|$ and $[D]=\sum_{\ell} a_{\ell}\left[D_{\ell}\right]$ for some integers $a_{\ell}$. Since $\hat{s}\left(N_{\mathcal{J}} X\right)$ and $\hat{\omega}$ are smooth it follows that

$$
\mathbf{1}_{Z_{\ell} \hat{s}}\left(N_{\mathcal{J}} X\right)=\pi_{*} \mathbf{1}_{j^{-1} D_{\ell}}\left(\frac{1}{1-j^{*} \hat{\omega}}\right) .
$$

Multiplying by $a_{\ell}$ and applying $i_{*}$ to the left hand side of (7.9) we get

$$
\hat{s}\left(N_{\mathcal{J}} X\right) \wedge a_{\ell}\left[Z_{\ell}\right]
$$

The same action on the right hand side of (7.9) gives, using that $i_{*} \pi_{*}=p_{*} j_{*}$,

$$
p_{*}\left(a_{\ell}\left[D_{\ell}\right] \wedge \frac{1}{1-\hat{\omega}}\right) .
$$

Summing up we get

$$
\hat{s}\left(N_{\mathcal{J}} X\right) \wedge \sum_{\ell} a_{\ell}\left[Z_{\ell}\right]=p_{*}\left([D] \wedge \frac{1}{1-\hat{\omega}}\right)=p_{*}\left(\sum_{k}[D] \wedge \hat{\omega}^{k}\right)=M^{\varphi},
$$

where the last equality follows from (5.9) (with $W=B l_{\mathcal{J}} X$ and $\alpha=1$ ); notice that $M_{0}^{\varphi}=0$ here. It remains to see that $\sum_{\ell} a_{\ell}\left[Z_{\ell}\right]$ is the fundamental cycle $\left[Z_{\mathcal{J}}\right]$ : Since $\hat{s}_{0}\left(N_{\mathcal{J}} X\right)=1$ we get from (7.10) that $M_{\kappa}^{\varphi}=\sum_{\ell} a_{\ell}\left[Z_{\ell}\right]$. The same argument applied to the section $s=\left(s_{1}, \ldots, s_{\kappa}\right)$ of the trivial rank $\kappa$-bundle (with trivial metric) over $U$ 
gives that $M_{\kappa}^{s}=\sum_{\ell} a_{\ell}\left[Z_{\ell}\right]$. It follows from [10, Ch. 3.16, Thm 3] that $M^{s}=M_{\kappa}^{s}$ is the proper intersection $\left[\operatorname{div} s_{1}\right] \wedge \cdots \wedge\left[\operatorname{div} s_{\kappa}\right]$, and it follows from $[12, C h .7]$ that this product is the fundamental cycle $\left[Z_{\mathcal{J}}\right]$ in case of a regular embedding.

Remark 7.4 In the proof above we did not describe $\tilde{j}$ explicitly. With the notation above, in a set $p^{-1} U$ we can consider $L_{D} \rightarrow B l_{\mathcal{J}} U$ as the line subbundle of $B l_{\mathcal{J}} U \times$ $\mathbb{C}^{\kappa}$ such that the fibre over a point $(x,[t])$ is the line $\left\{\lambda t \in \mathbb{C}^{\kappa} ; \lambda \in \mathbb{C}\right\}$. Thus $\tilde{j}$ maps the point $(x,[\xi(s)], \xi(s)]$ to $(x,[t], t)$ in $L_{D}$.

It is well-known, and indeed follows from the proof above, that $B l_{\mathcal{J}} X$ can be seen as the closure in $\mathbb{P}(E)$ of the graph $\{(x,[\varphi(x)]) \in \mathbb{P}(E) ; x \in X \backslash Z\}$; then the mapping $\psi$ is of course just the natural inclusion.

Corollary 7.5 Let $i: V \hookrightarrow X$ be an irreducible subvariety and assume that $i^{*} \varphi$ defines a regular embedding of codimension $\kappa$ in $V$. Then

$$
M^{\varphi} \wedge[V]=\hat{s}\left(N_{\mathcal{J}} X\right) \wedge\left[Z_{\mathcal{J}}\right] \wedge[V]
$$

Proof From Proposition 1.5 we have for degree reasons that $M_{\mathcal{K}}^{\varphi}=\left[Z_{\mathcal{J}}\right]$. Since $Z$ and $V$ intersect properly by assumption, $\left[Z_{\mathcal{J}}\right] \wedge[V]$ makes sense and, moreover, $M_{\kappa}^{\varphi} \wedge[V]=\left[Z_{\mathcal{J}}\right] \wedge[V]$, cf. [5, Section 2.4] and (5.15). On the other hand, from Proposition 1.5 applied to $i^{*} \mathcal{J}$, cf. (5.12), $M_{\kappa}^{\varphi} \wedge[V]=i_{*} M_{\kappa}^{i^{*} \varphi}=i_{*}\left[Z_{i^{*}} \mathcal{J}\right]$. Thus

$$
i_{*}\left[Z_{i^{*} \mathcal{J}}\right]=\left[Z_{\mathcal{J}}\right] \wedge[V] .
$$

If the tuple $s=\left(s_{1}, \ldots, s_{\kappa}\right)$ generates $\mathcal{J}$, then $i^{*} s$ generates $i^{*} \mathcal{J}$ and the transition matrices of $N_{i * \mathcal{J}} V$ are the restriction to $V$ of the transition matrices of $N_{\mathcal{J}} X$. Thus $N_{i^{*} \mathcal{J}} V=i^{*} N_{\mathcal{J}} X$. Moreover, the Hermitian metric on $N_{i * \mathcal{J}} V$ is inherited from $N_{\mathcal{J}} X$ so that

$$
\hat{s}\left(N_{i * \mathcal{J}} V\right)=i^{*} \hat{s}\left(N_{\mathcal{J}} X\right) .
$$

By Proposition 1.5, (5.12), (7.11) and (7.12) we thus get

$$
\begin{aligned}
M^{\varphi} \wedge[V] & =i_{*} M^{i^{*} \varphi}=i_{*}\left(\hat{s}\left(N_{i^{*} \mathcal{J}} V\right) \wedge\left[Z_{i^{*} \mathcal{J}}\right]\right)=i_{*}\left(i^{*} \hat{s}\left(N_{\mathcal{J}} X\right) \wedge\left[Z_{i^{*} \mathcal{J}}\right]\right) \\
& =\hat{s}\left(N_{\mathcal{J}} X\right) \wedge\left[Z_{\mathcal{J}}\right] \wedge[V] .
\end{aligned}
$$

Proof of Proposition 1.4 Notice that the right-hand side of the equation in the formulation of the proposition is well-defined in view of Proposition 4.1. In view of Theorem 1.2 and (4.4) the proposition follows immediately from Proposition 1.5 if there is a vector bundle with a section defining $\mathcal{J}$. If not we still have, cf. Remark 7.4, the commutative diagram 


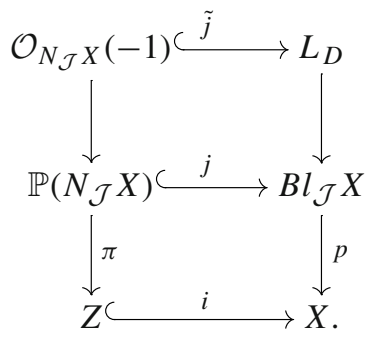

By definition, cf. (1.1), recalling that $\kappa \geq 1, S(\mathcal{J}, X)=p_{*}\left([D] \wedge 1 /\left(1+c_{1}\left(L_{D}\right)\right)\right)$ and, by (2.4), $s\left(N_{\mathcal{J}} X\right)=\pi_{*}\left(1 /\left(1+j^{*} c_{1}\left(L_{D}\right)\right)\right)$. Thus the result follows as in the proof of Proposition 1.5, replacing computations in $\mathcal{G} \mathcal{Z}(X)$ by analogous ones in $\mathcal{B}(X)$.

Proposition 7.6 Let $\sigma$ be a holomorphic section of a Hermitian bundle $E \rightarrow X$ defining the regular embedding $\mathcal{J}$ and let $\varphi$ be a holomorphic section of a Hermitian bundle $F \rightarrow X$ defining a regular embedding of codimension 1 . Suppose that the section $\sigma+\varphi$ of the Hermitian bundle $E \oplus F \rightarrow X$ defines a regular embedding of codimension $\kappa+1$. Then

$$
M^{\sigma+\varphi}=M^{\sigma} \wedge M^{\varphi}=M^{\varphi} \wedge M^{\sigma}
$$

Proof Let us first assume that $\kappa=1$. Then the statement is symmetric in $\sigma$ and $\varphi$; $\sigma$ and $\varphi$ are sections of line subbundles $L_{1} \subset E$ and $L_{2} \subset F$ defining divisors $D_{1}$ and $D_{2}$, respectively. By Proposition 1.5, $M^{\varphi}=\hat{s}\left(L_{2}\right) \wedge\left[D_{2}\right]$, cf. (7.5), and so, by Corollary 7.5, since $\left.\sigma\right|_{\left|D_{2}\right|}$ is generically non-vanishing,

$$
M^{\sigma} \wedge M^{\varphi}=\hat{s}\left(L_{1}\right) \wedge \hat{s}\left(L_{2}\right) \wedge\left[D_{1}\right] \wedge\left[D_{2}\right]
$$

We have that $\sigma+\varphi$ is a section of the Hermitian bundle $\mathcal{E}:=L_{1} \oplus L_{2} \subset E \oplus F$ defining a regular embedding of codimension 2 . Denote the corresponding ideal by $\mathcal{J}^{\prime}$, its zero set by $Z^{\prime}$, and notice that $N_{\mathcal{J}^{\prime}} X=\left.\mathcal{E}\right|_{Z^{\prime}}$. By Proposition 1.5 we have

$$
M^{\sigma+\varphi}=\hat{s}(\mathcal{E}) \wedge\left[Z_{\mathcal{J}^{\prime}}\right]=\hat{s}(\mathcal{E}) \wedge\left[D_{1}\right] \wedge\left[D_{2}\right]
$$

where the last equality follows as in the end of the proof of Proposition 1.5. It follows from (2.7) and (2.10) that $\hat{s}(\mathcal{E})=\hat{s}\left(L_{1}\right) \wedge \hat{s}\left(L_{2}\right)$. This concludes the proof when $\kappa=1$.

Now assume that $\kappa \geq 2$. Let $p: B l_{\mathcal{J}} X \rightarrow X$ be the blow-up of $X$ along $\mathcal{J}$. Then both $p^{*} \sigma$ and $p^{*} \varphi$ define principal ideals and it is readily verified that $p^{*} \sigma+p^{*} \varphi$ defines a regular embedding in $B l_{\mathcal{J}} X$ of codimension 2. Since $p$ is a modification it is generically an isomorphism and hence from Example 5.3, Theorem 5.2 (iv), and the case $\kappa=1$ proved above, we get

$$
M^{\sigma+\varphi}=p_{*} M^{p^{*} \sigma+p^{*} \varphi}=p_{*}\left(M^{p^{*} \sigma} \wedge M^{p^{*} \varphi}\right)=M^{\sigma} \wedge p_{*}\left(M^{p^{*} \varphi}\right)=M^{\sigma} \wedge M^{\varphi} .
$$


Remark 7.7 It is not necessary to assume that $\sigma$ defines a regular embedding; the proof only relies on the fact that $p^{*} \sigma+p^{*} \varphi$ defines a regular embedding. One can therefore formulate a variant of Proposition 7.6 that is a global version of Lemma 9.2 in $[5]$.

Example 7.8 Let $\tau: X^{\prime} \rightarrow X$ be a section of a locally trivial fibration $\pi: X \rightarrow X^{\prime}$ with one-dimensional fibers, let $\varphi$ be a section of a Hermitian line bundle $L \rightarrow X$ defining $\tau\left(X^{\prime}\right)$, and let $\sigma^{\prime}$ be a section of a Hermitian bundle $E^{\prime} \rightarrow X^{\prime}$ defining a regular embedding. If $\sigma=\pi^{*} \sigma^{\prime}$, then

$$
\tau_{*} M^{\sigma^{\prime}}=\hat{c}(L) \wedge M^{\sigma+\varphi} .
$$

To see this, notice first that it follows from Proposition 1.5 and (2.7) that $\hat{c}(L) \wedge M^{\varphi}=$ $[\varphi=0]=\left[\tau\left(X^{\prime}\right)\right]$. Thus by Proposition 7.6, $\hat{c}(L) \wedge M^{\sigma+\varphi}=M^{\pi^{*} \sigma^{\prime}} \wedge \hat{c}(L) \wedge M^{\varphi}=$ $M^{\pi^{*} \sigma^{\prime}} \wedge\left[\tau\left(X^{\prime}\right)\right]=\tau_{*} M^{\sigma^{\prime}}$.

Let $i: Z_{\mathcal{J}} \hookrightarrow X$ be a regular embedding of codimension $\kappa \geq 1$. We conclude with a short discussion of the $\mathcal{B}$-Gysin mapping (1.8). It is further studied in [7]. In analogy with Chow theory, cf. [12, Ch. 6], one can think of $\left(c\left(N_{\mathcal{J}} X\right) \wedge S(\mathcal{J}, X) \wedge \mu\right)_{k-\kappa}$ as an intersection of $Z_{\mathcal{J}}$ and $\mu$ in $\mathcal{B}(X)$. We assume that $\mathcal{J}$ is defined by the section $\varphi$ of the Hermitian bundle $F \rightarrow X$ so we can also consider the more explicit mapping (1.9).

First, let $\gamma \in \mathcal{G Z}_{k}(X)$ be a product of components of Chern or Segre forms. We claim that

$$
\left(\hat{c}\left(N_{\mathcal{J}} X\right) \wedge M^{\varphi} \wedge \gamma\right)_{k-\kappa}=\left[Z_{\mathcal{J}}\right] \wedge \gamma=i_{*} i^{*} \gamma,
$$

so that (1.9) can be seen as a generalization to $\mathcal{G} \mathcal{Z}(X)$ of $i_{*} i^{*}$. In fact, by (5.14), $M^{\varphi} \wedge \gamma=\gamma \wedge M^{\varphi}$, and so (7.14) follows from Proposition 1.5 and (2.7). In the same way (1.8) is a generalization to $\mathcal{B}(X)$ of $i_{*} i^{*}$.

Example 7.9 If $Z_{\mathcal{J}}$ is a divisor, i.e., $\kappa=1$, then we can assume that $\varphi$ is a section of a line bundle $L \rightarrow X$. Then $N_{\mathcal{J}} X=\left.L\right|_{Z}$, cf. (7.5). Assume that $\mu \in \mathcal{G Z}_{k}(X)$ is irreducible. If $\varphi$ vanishes identically on $\mu$, then $M^{\varphi} \wedge \mu=\mu$, and hence $\left(\hat{c}(L) \wedge M^{\varphi} \wedge \mu\right)_{k-1}=\hat{c}_{1}(L) \wedge \mu$. Otherwise $M_{0}^{\varphi} \wedge \mu=0$ and then

$$
\left(\hat{c}(L) \wedge M^{\varphi} \wedge \mu\right)_{k-1}=M_{1}^{\varphi} \wedge \mu .
$$

\section{Variants of the Poincaré-Lelong formula}

Let $h$ be a meromorphic section of a Hermitian line bundle $L \rightarrow X$. We say the div $h$ intersects $\mu \in \mathcal{G Z}_{k}(X)$ properly if for each irreducible component $\mu_{j}$ of $\mu$, divh intersects $\left|\mu_{j}\right|$ properly, cf. Sect. 2 , i.e., $h$ is non-trivial on each $\left|\mu_{j}\right|$. We have the following Poincaré-Lelong formula "on $\mu$ ". 
Proposition 8.1 Assume that $h$ is a meromorphic section of $L \rightarrow X$ such that divh intersects $\mu \in \mathcal{G Z}_{k}(X)$ properly. Then $\log |h|^{2} \cdot \mu$, a priori defined where $h$ is holomorphic and non-zero, extends to a current of order 0 on $|\mu|$. Moreover, there is a generalized cycle $[\operatorname{div} h] \wedge \mu$ in $\mathcal{G Z}_{k-1}(X)$ with Zariski support on $|\operatorname{div} h| \cap|\mu|$ such that

$$
d d^{c}\left(\log |h|^{2} \cdot \mu\right)=[\operatorname{div} h] \wedge \mu-\hat{c}_{1}(L) \wedge \mu .
$$

If $\mu \sim 0$, then $[\operatorname{divh}] \wedge \mu \sim 0$.

We say that $[\operatorname{div} h] \wedge \mu$ is the proper intersection of div $h$ and $\mu$. Choosing a trivial metric on $L$ locally, we see that $[\operatorname{div} h] \wedge \mu$ only depends on the divisor div $h$ and not on $h$ (since $d d^{c}(u \cdot \mu)=0$ if $u$ is pluriharmonic). In view of the last statement of the proposition, [div $h$ ] $\wedge \mu$ is well-defined in $\mathcal{B}_{k-1}(X)$ for $\mu \in \mathcal{B}_{k}(X)$.

Proof By assumption, $\log |h|^{2} \cdot \mu$ is generically defined on $|\mu|$. Each irreducible component $\mu_{j}$ of $\mu$ is a finite sum of non-zero generalized cycles $\mu^{\prime}=\tau_{*} \alpha$ with $\tau(W)=V:=\left|\mu_{j}\right|$, see Remark 3.10 and Lemma 3.8. Let us consider such a $\mu^{\prime}$ and let $V^{\prime} \subset V$ be a subset of positive codimension such that $h$ is holomorphic and non-vanishing on $V \backslash V^{\prime}$. Then

$$
\log |h|^{2} \cdot \mu^{\prime}=\tau_{*}\left(\log \left|\tau^{*} h\right|^{2} \cdot \alpha\right)
$$

holds there, and since the right hand side has an extension to $V$ of order 0 so has the left hand side. Since $\tau^{-1} V^{\prime}$ has positive codimension in $W, \mathbf{1}_{V^{\prime}} \tau_{*}\left(\log \left|\tau^{*}\right|^{2} \cdot \alpha\right)=$ $\tau_{*}\left(\mathbf{1}_{\tau^{-1} V^{\prime}}\left(\log \left|\tau^{*}\right|^{2} \cdot \alpha\right)\right)=0$. Summing up the first claim of the proposition follows.

Consider again a $\mu^{\prime}=\tau_{*} \alpha$ as above. From the usual Poincaré-Lelong formula, cf. Proposition 2.1, we have

$$
d d^{c}\left(\log \left|\tau^{*} h\right|^{2} \cdot \alpha\right)=\left[\operatorname{div} \tau^{*} h\right] \wedge \alpha-\hat{c}_{1}\left(\tau^{*} L\right) \wedge \alpha
$$

on $W$. Summing up we get (8.1) with $[\operatorname{div} h] \wedge \mu$ defined as the sum of all $\tau_{*}\left(\left[\operatorname{div} \tau^{*} h\right] \wedge \alpha\right)$. The last statement of the proposition follows since $\tau_{*}\left(\left[\operatorname{div} \tau^{*} h\right] \wedge \alpha\right) \sim$ 0 if $\alpha$ is of the form $\beta \wedge \alpha^{\prime}$, where $\beta$ is a component of a $B$-form.

If $h$ is holomorphic, $M_{1}^{h} \wedge \mu=\tau_{*}\left(M_{1}^{\tau^{*} h} \wedge \alpha\right)=\tau_{*}\left(\left[\operatorname{div} \tau^{*} h\right] \wedge \alpha\right)$ and thus, cf. (7.15),

$$
[\operatorname{div} h] \wedge \mu=M_{1}^{h} \wedge \mu
$$

It follows as in the proof of Theorem 5.2 that $\log |h|^{2} \cdot \mu=\lim _{\epsilon} \log \left(|h|^{2}+\epsilon\right) \cdot \mu$.

Now assume that $h=\left(h_{1}, \ldots, h_{m}\right)$ is a tuple of global sections of $L$ and consider the section $h$ of $\oplus_{1}^{m} L$. In view of (5.2) we have

$$
d d^{c}\left(\log |h|^{2} \cdot \mu\right)=d d^{c} \log |h|^{2} \wedge \mu
$$

where the right hand side is defined by the limit procedure in (5.2). If $e$ is a local frame for $L$, then $h=h(e) e$, where $h(e)$ is a tuple of holomorphic functions. Clearly 
$\log |h(e)|^{2}$ depends on the choice of frame but $d d^{c} \log |h(e)|^{2}$ does not. Thus

$$
d d^{c} \log |h|_{0}^{2} \wedge \mu:=d d^{c} \log |h(e)|^{2} \wedge \mu
$$

is a well-defined global current which in addition is independent of the Hermitian metric on $L$.

Remark 8.2 Let $U \subset X$ be an open set where we have a local frame $e$ for $L$. If we choose the metric on $L$ in $U$ so that $|e|=1$ and equip $E=\oplus_{0}^{m} L$ with the induced metric, then $d d^{c} \log |\sigma|_{\circ}^{2} \wedge \mu=d d^{c} \log |\sigma|^{2} \wedge \mu$.

For instance, if $h$ is just one single section, i.e., $m=1$, then (8.1) implies that $d d^{c}\left(\log |h|_{0}^{2} \cdot \mu\right)=[\operatorname{div} h] \wedge \mu$.

Example 8.3 Let $\theta=d d^{c} \log \left(\left|z_{1}\right|^{2}+\left|z_{2}\right|^{2}\right)$ be the generalized cycle in $\mathbb{P}^{2}$ of Example 3.11 and let $\sigma$ be a section of $\mathcal{O}(1)$ defining a line through $p=[1: 0: 0]$. Then $\theta$ has dimension 1 , it is irreducible and $|\theta|=\mathbb{P}^{2}$. Thus $\operatorname{div} \sigma$ intersects $\theta$ properly. We claim that $[\operatorname{div} \sigma] \wedge \theta=[p]$. Let $i: V \hookrightarrow \mathbb{P}^{2}$ be the line $\operatorname{div} \sigma$. Notice that if we consider $z_{j}$ as sections of the line bundle $\mathcal{O}(1) \rightarrow \mathbb{P}^{2}$, then $\theta=d d^{c} \log |h|_{0}^{2}$, where $h=\left(z_{1}, z_{2}\right)$. Now

$$
[\operatorname{div} \sigma] \wedge \theta=\theta \wedge[\operatorname{div} \sigma]=d d^{c}\left(\log |h|_{\circ}^{2} \cdot[V]\right)=i_{*} d d^{c} \log \left|i^{*} h\right|_{\circ},
$$

where the first equality follows from [11, Ch. III Corollary 4.11] and the second one from (8.5). In the affinization where $z_{0}=1$ we have the frame element $e=z_{0}$, so in local coordinates $\left(z_{1}, z_{2}\right)$ we have $\log |h(e)|^{2}=\log \left(\left|z_{1}\right|^{2}+\left|z_{2}\right|^{2}\right)$; notice that it is harmonic on $V \backslash\{p\}$ and has a simple pole at $p$ so that $d d^{c} \log \left|i^{*} h\right|_{\circ}^{2}=[p]$. Now the claim follows since $i_{*}[p]=[p]$. Notice that $\operatorname{dim}|\theta|=2$ while $\operatorname{dim} \mid[\operatorname{div} h] \wedge$ $\theta \mid=0$.

\section{The $\mathcal{B}$-Stückrad-Vogel class}

Throughout this section $X$ is a compact (reduced) analytic space and $\mathcal{J} \rightarrow X$ is generated by a finite number of global sections of the line bundle $L \rightarrow X$, to begin with without any specified Hermitian metric. For instance, if $X$ is projective, then given $\mathcal{J} \rightarrow X$ there is a very ample $L \rightarrow X$ such that $L \otimes \mathcal{J}$ is globally finitely generated, see, e.g., [18, Theorem 1.2.6].

The classical Stückrad-Vogel (SV) algorithm, [20], is a way to produce intersections by reducing to proper intersections of cycles by divisors. The resulting $\mathrm{SV}$-cycles define an element, the SV-class $v(\mathcal{J}, L, X)$, in $\mathcal{A}(Z)$ that only depends on $\mathcal{J}$ and the line bundle $L$. It is related to the Segre class $s(\mathcal{J}, X)$ via van Gastel's formulas, [14], see below.

We shall define an analogous $\mathcal{B}$-SV class $V(\mathcal{J}, L, \mu)$ in $\mathcal{B}(|\mu| \cap Z)$ for any $\mu \in$ $\mathcal{B}(X)$, and this class will be related to our Segre class $S(\mathcal{J}, \mu)$ via analogues of van Gastel's formulas. To motivate our definition we first consider the SV-algorithm on a generalized cycle $\mu \in \mathcal{G Z}_{d}(X)$ : If $\mu_{0}:=\mathbf{1}_{X \backslash Z} \mu=0$ then $\mathcal{J}$ vanishes identically 
on $\mu$ and the algorithm stops directly. Otherwise, let $\mu^{1}, \ldots, \mu^{m}$ be the irreducible components of $\mu_{0}$. These are precisely the irreducible components of $\mu$ that are not contained in $Z$. For each $j$ the set of $h \in \Gamma(X, L \otimes \mathcal{J})$ that vanish identically on $\mu^{j}$ is a proper subspace $V^{j}$ of the finite-dimensional vector space $\Gamma(X, L \otimes \mathcal{J})$. Thus each $h \in \Gamma(X, L \otimes \mathcal{J})$ in the complement of $\cup_{j} V^{j}$ intersects $\mu_{0}$ properly, by definition; that is, a generic $h$ will do. Let us choose such a section and call it $h_{1}$. Next, we consider $\mu_{1}:=\mathbf{1}_{X \backslash Z}\left[\operatorname{div} h_{1}\right] \wedge \mu_{0}$. If $\mu_{1}$ is empty the algorithm stops. If not, a generic $h$ intersects $\mu_{1}$ properly. Let us choose such a section and call it $h_{2}$. We proceed in this way until $\mu_{k}=0$ for some $k \leq d$ and the algorithm stops. If $\mu_{d}:=\mathbf{1}_{X \backslash Z}\left[\operatorname{div} h_{d}\right] \wedge \mu_{d-1}$ is nonempty, then since $\mu_{d}$ has dimension 0 , any proper intersection with a divisor div $h$ will give just 0 , and the $\mathrm{SV}$-algorithm stops.

If $\mu_{k}=0$ for some $k<d$, then we can choose $h_{k+1}, \ldots, h_{d}$ in an arbitrary way if we adopt the convention that any div $h$ intersects the generalized cycle 0 properly and $[\operatorname{div} h] \cdot 0=0$. We have the following definitions:

Definition 9.1 An ordered sequence $h=\left(h_{1}, h_{2}, \ldots, h_{d}\right)$ of sections of $L \otimes \mathcal{J}$ is a Stückrad-Vogel (SV) sequence on $\mu \in \mathcal{G Z}_{d}(X)$ if div $h_{k}$ intersects

$$
\mathbf{1}_{X \backslash Z}\left[\operatorname{div} h_{k-1}\right] \wedge \cdots \wedge \mathbf{1}_{X \backslash Z}\left[\operatorname{div} h_{1}\right] \wedge \mathbf{1}_{X \backslash Z \mu}
$$

properly, $k=1, \ldots, d$. Given a SV-sequence $h$ on $\mu$, we have the associated ${ }^{9} S V$-cycle

$$
v^{h} \wedge \mu=\sum_{k=0}^{d} v_{k}^{h} \wedge \mu,
$$

where

$$
v_{0}^{h} \wedge \mu:=\mathbf{1}_{Z} \mu, v_{k}^{h} \wedge \mu:=\mathbf{1}_{Z}\left[\operatorname{div} h_{k}\right] \wedge \mathbf{1}_{X \backslash Z}\left[\operatorname{div} h_{k-1}\right] \wedge \cdots \wedge \mathbf{1}_{X \backslash Z}\left[\operatorname{div} h_{1}\right] \wedge \mathbf{1}_{X \backslash Z} \mu, k \geq 1
$$

Here we use the convention that $\mathbf{1}_{V}$ acts on the whole current on its right, i.e., $\mathbf{1}_{V} a \wedge b \wedge \ldots=\mathbf{1}_{V}(a \wedge b \wedge \ldots)$, cf. [5, Sections 3 and 6].

Example 9.2 If $\mathcal{J}$ vanishes identically on $\mu$, then $v^{h}=v_{0}^{h}=\mu$.

If div $h$ does not intersect $\mu$ properly we can still define [div $h] \wedge \mu$ by (8.4). Since $M_{1}^{h} \wedge \mu^{\prime}=0$ if $h$ vanishes identically on $\left|\mu^{\prime}\right|$ we have that $[\operatorname{div} h] \wedge \mu=\sum_{j}[\operatorname{div} h] \wedge \mu_{j}^{\prime}$, where $\mu_{j}^{\prime}$ are the irreducible components of $\mu$ that divh intersects properly. By this convention therefore $[\operatorname{div} h] \wedge \mu=[\operatorname{div} h] \wedge \mathbf{1}_{X \backslash Z} \mu$ if the right hand side is a proper intersection. For any sequence $h=\left(h_{1}, \ldots, h_{d}\right)$ of sections of $L \otimes \mathcal{J}$ we can thus define (9.2) with

$$
v_{0}^{h} \wedge \mu=\mathbf{1}_{Z} \mu, \quad v_{k}^{h} \wedge \mu=\mathbf{1}_{Z}\left[\operatorname{div} h_{k}\right] \wedge \mu \cdots \wedge\left[\operatorname{div} h_{1}\right] \wedge \mu, k=1,2, \ldots d,
$$

and as long as $h$ is a $\mathrm{SV}$-sequence it is consistent with the previous definition.

\footnotetext{
9 It would me more correct but somewhat inconvenient to use the term SV-generalized cycle.
} 
Let $\sigma=\left(\sigma_{0}, \ldots, \sigma_{m}\right)$ be a sequence of global sections of $L$ that generate $\mathcal{J}$. Given $a \in \mathbb{P}^{m}, a=\left[a_{0}: \cdots: a_{m}\right]$, let $a \cdot \sigma=a_{0} \sigma_{0}+\cdots+a_{m} \sigma_{m}$ which is welldefined up to a nonzero constant. If $a=\left(a_{1}, \ldots, a_{d}\right) \in\left(\mathbb{P}^{m}\right)^{d}$ is a generic tuple, then $a \cdot \sigma=\left(a_{1} \cdot \sigma, a_{2} \cdot \sigma, \ldots, a_{d} \cdot \sigma\right)$ is a SV-sequence on $\mu$ and, cf. (9.3),

$$
v^{a \cdot \sigma} \wedge \mu=\mathbf{1}_{Z} \mu+\sum_{k \geq 1} \mathbf{1}_{Z}\left[\operatorname{div}\left(a_{k} \cdot \sigma\right)\right] \wedge \cdots \wedge\left[\operatorname{div}\left(a_{1} \cdot \sigma\right)\right] \wedge \mu
$$

is the associated SV-cycle. As observed above, however, (9.4) makes sense for any $\left(a_{1}, \ldots, a_{d}\right) \in\left(\mathbb{P}^{m}\right)^{d}$.

Proposition 9.3 Assume that $\mu \in \mathcal{G Z}(X)$. Then

$$
\int_{\left(\mathbb{P}^{m}\right)^{k}}\left[\operatorname{div}\left(a_{k} \cdot \sigma\right)\right] \wedge \cdots \wedge\left[\operatorname{div}\left(a_{1} \cdot \sigma\right)\right] \wedge \mu d V(a)=\left(d d^{c} \log |\sigma|_{\circ}^{2}\right)^{k} \wedge \mu, \quad k=1,2, \ldots,
$$

where $d V(a)=\wedge_{j=1}^{k}\left(d d^{c} \log \left|a_{j}\right|^{2}\right)^{m}$ is the natural normalized volume form on $\left(\mathbb{P}^{m}\right)^{k}$.

Proof We may assume that $\mu=\tau_{*} \alpha$ where $\tau: W \rightarrow X$ and $W$ is connected. Then $\mu$ is irreducible. If $\mathcal{J}$ vanishes identically on $\mu$, then $\sigma \equiv 0$ on $\mu$ and by definition both sides of (9.5) vanish. Thus we can assume that $\tau^{*} \mathcal{J}$ is nontrivial on $W, \tau^{*} \mathcal{J}$ is principal and that the exceptional divisor $D$ is defined by the section $\sigma^{0}$ of the line bundle $L_{D} \rightarrow W$. Then $\tau^{*} \sigma=\sigma^{0} \sigma^{\prime}$ where $\sigma^{\prime}=\left(\sigma_{0}^{\prime}, \ldots, \sigma_{m}^{\prime}\right)$ is a non-vanishing tuple of sections of $L_{D}^{-1} \otimes \tau^{*} L$. Notice that

$$
d d^{c} \log \left|a_{j} \cdot \tau^{*} \sigma\right|_{\circ}^{2}=d d^{c} \log \left|\sigma^{0}\right|_{\circ}^{2}+d d^{c} \log \left|a_{j} \cdot \sigma^{\prime}\right|_{\circ}^{2}=[D]+\left[\operatorname{div}\left(a_{j} \cdot \sigma^{\prime}\right)\right] .
$$

Since $\sigma^{\prime}$ is non-vanishing on $|D|$, as in [5, Eq. (6.3)], for generic $a_{j}$ we have

$$
\begin{aligned}
& {\left[\operatorname{div}\left(a_{k} \cdot \sigma\right)\right] \wedge \cdots \wedge\left[\operatorname{div}\left(a_{1} \cdot \sigma\right)\right] \wedge \mu} \\
& =\tau_{*}\left([D] \wedge\left[\operatorname{div}\left(a_{k-1} \cdot \sigma^{\prime}\right)\right] \wedge \cdots \wedge\left[\operatorname{div}\left(a_{1} \cdot \sigma^{\prime}\right)\right] \wedge \alpha\right. \\
& \left.\quad+\left[\operatorname{div}\left(a_{k} \cdot \sigma^{\prime}\right)\right] \wedge \cdots \wedge\left[\operatorname{div}\left(a_{1} \cdot \sigma^{\prime}\right)\right] \wedge \alpha\right),
\end{aligned}
$$

where all intersections are proper. By [5, Lemma 6.3] the left hand side of (9.5) is therefore equal to

$$
\tau_{*}\left([D] \wedge\left(d d^{c} \log \left|\sigma^{\prime}\right|_{\circ}^{2}\right)^{k-1} \wedge \alpha+\left(d d^{c} \log \left|\sigma^{\prime}\right|_{\circ}^{2}\right)^{k} \wedge \alpha\right) .
$$

Now assume that we locally have a flat metric on $L$. With the notation in the proof of Theorem 5.2 we then have $d d^{c} \log \left|\sigma^{\prime}\right|_{\circ}^{2}=\hat{\omega}$ since $\sigma^{\prime}$ is a tuple of sections of $L_{D}^{-1}$ and $\left|\sigma^{\prime}\right|=1$, cf. (9.12) below. From (5.8) we can therefore deduce that (9.6) is equal to the right hand side of $(9.5)$.

Remark 9.4 Proposition 9.3 is similar to [5, Theorem 6.2]. The analogues of the identities (6.8) and (6.9) in that theorem hold in the present situation as well; after adaption to the present situation the proof in [5] goes through. 
Definition 9.5 Given a line bundle $L$ and a tuple $\sigma=\left(\sigma_{0}, \ldots, \sigma_{m}\right)$ of sections of $L$ that generate $\mathcal{J}$ and $\mu \in \mathcal{G} \mathcal{Z}(X)$ we define the generalized cycle

$$
M^{L, \sigma} \wedge \mu=\mathbf{1}_{Z} \mu+\sum_{k \geq 1} \mathbf{1}_{Z}\left(d d^{c} \log |\sigma|_{\circ}^{2}\right)^{k} \wedge \mu .
$$

It follows from (9.4) and (9.5) that $M^{L, \sigma} \wedge \mu$ is a mean value of SV-cycles on $\mu$.

Notice that in general, the subspace of $\Gamma(X, L)$ generated by $\sigma_{0}, \ldots, \sigma_{m}$ is proper. Nevertheless, the class of $M^{L, \sigma} \wedge \mu$ in $\mathcal{B}(X)$ is independent of the choice of tuple $\sigma$ :

Proposition 9.6 If $g$ is another tuple of sections of L that generate $\mathcal{J}, \mu, \mu^{\prime} \in \mathcal{G} \mathcal{Z}(X)$ and $\mu^{\prime} \sim \mu$, then $M^{L, \sigma} \wedge \mu \sim M^{L, g} \wedge \mu^{\prime}$.

Proof We first consider $\mu$ and keep the notation from the proof of Proposition 9.3. If $\mathcal{J}$ vanishes on $|\mu|$, then $M^{L, \sigma} \wedge \mu=M_{0}^{L, \sigma} \wedge \mu=\mu$. Thus we assume that $\tau^{*} \sigma=\sigma^{0} \sigma^{\prime}$ on $W$ as usual. Since $\sigma^{\prime}$ is a non-vanishing tuple of sections of $L_{D}^{-1} \otimes \tau^{*} L, 1 /\left|\sigma^{\prime}\right|_{\circ}^{2}$ is a metric on $L_{D}^{-1} \otimes \tau^{*} L$ and hence $d d^{c} \log \left|\sigma^{\prime}\right|_{0}^{2}$ is a representative of the first Chern class $c_{1}\left(L_{D}^{-1} \otimes \tau^{*} L\right)$. From (9.6) and (2.2) we have

$$
M_{k}^{L, \sigma} \wedge \mu=\mathbf{1}_{Z}\left(d d^{c} \log |\sigma|_{\circ}^{2}\right)^{k} \wedge \mu=\tau_{*}\left([D] \wedge\left(d d^{c} \log \left|\sigma^{\prime}\right|_{\circ}^{2}\right)^{k-1} \wedge \alpha\right), \quad k=1,2, \ldots
$$

Now $\tau^{*} g=\sigma^{0} g^{\prime}$, where $g^{\prime}$ also is a tuple of sections of $L_{D}^{-1} \otimes \tau^{*} L$, and hence $d d^{c} \log \left|g^{\prime}\right|_{0}^{2}$ and $d d^{c} \log \left|\sigma^{\prime}\right|_{0}^{2}$ differ by a $B$-form; in fact the difference is $d d^{c}$ of the global function $\log \left(\left|g^{\prime}\right|_{0}^{2} /\left|\sigma^{\prime}\right|_{o}^{2}\right)$. Thus the class in $\mathcal{B}(X)$ is independent of the choice of tuple. Finally, if $\beta$ is a component of a $B$-form and $\alpha=\beta \wedge \alpha^{\prime}$, then (9.7) is $\sim 0$ in $\mathcal{G} \mathcal{Z}(X)$.

In view of Remark 8.2 we have that $M^{L, \sigma} \wedge \mu=M^{\sigma} \wedge \mu$ in $U \subset X$ for a suitable metric on $L$ on $U$. Therefore local statements that hold for $M^{\sigma} \wedge \mu$ must hold for $M^{L, \sigma} \wedge \mu$ as well: For instance, if $\gamma$ is a component of a Chern or Segre form, then by (5.14),

$$
M^{L, \sigma} \wedge(\gamma \wedge \mu)=\gamma \wedge M^{L, \sigma} \wedge \mu \text {. }
$$

If $h: X^{\prime} \rightarrow X$ is proper and $\sigma$ is a tuple of sections of $L$ that generate $\mathcal{J} \rightarrow X$, then $h^{*} \sigma$ is a tuple of sections of $h^{*} L$ that generate $h^{*} \mathcal{J} \rightarrow X^{\prime}$. If $\mu \in \mathcal{G Z}\left(X^{\prime}\right)$, then by Theorem 5.2 (iv),

$$
h_{*}\left(M^{h^{*} L, h^{*} \sigma} \wedge \mu^{\prime}\right)=M^{L, \sigma} \wedge h_{*} \mu^{\prime}
$$

In view of Proposition 9.6 the following definition makes sense.

Definition 9.7 Assume that $L \rightarrow X$ has sections that generate $\mathcal{J}$ globally. For $\mu \in$ $\mathcal{B}(X)$ we let $V(\mathcal{J}, L, \mu)$, the $\mathcal{B}$-SV class, be the class in $\mathcal{B}(Z \cap|\mu|)$ defined by $M^{L, \sigma} \wedge \hat{\mu}$ for a tuple of generators $\sigma$ and a representative $\hat{\mu} \in \mathcal{G} \mathcal{Z}(X)$ of $\mu$.

We now relate the $\mathcal{B}$-SV class to the $\mathcal{B}$-Segre class in analogy with van Gastel's formulas [14], see (9.22) below. To this end we first give a $\mathcal{G Z}$-variant and therefore choose a Hermitian metric. 
Theorem 9.8 Let $\sigma=\left(\sigma_{0}, \ldots, \sigma_{m}\right)$ be a tuple of sections of $L$ that generate $\mathcal{J}$. Assume that we have a Hermitian metric on $L$ with first Chern form $\hat{\omega}_{L}$ and consider $\sigma$ as a section of the Hermitian vector bundle $E=\oplus_{0}^{m} L$. For $\mu \in \mathcal{G} \mathcal{Z}(X)$ we have

$$
M^{L, \sigma} \wedge \mu=\sum_{j \geq 0}\left(\frac{1}{1-\hat{\omega}_{L}}\right)^{j} \wedge M_{j}^{\sigma} \wedge \mu
$$

and

$$
M^{\sigma} \wedge \mu=\sum_{j \geq 0}\left(\frac{1}{1+\hat{\omega}_{L}}\right)^{j} \wedge M_{j}^{L, \sigma} \wedge \mu .
$$

Proof Let us assume that $\mu=\tau_{*} \alpha$ where $\tau: W \rightarrow X$ and $W$ is connected. If $\mathcal{J}$ vanishes identically on $\mu$ then $M^{\sigma} \wedge \mu=M_{0}^{\sigma} \wedge \mu=\mu$ and $M^{L, \sigma} \wedge \mu=M_{0}^{L, \sigma} \wedge \mu=\mu$ and thus (9.10) and (9.11) are both trivially true.

We can thus assume that $\tau^{*} \sigma=\sigma^{0} \sigma^{\prime}$, where $\sigma^{\prime}$ is a non-vanishing tuple of sections of $L_{D}^{-1} \otimes \tau^{*} L$, or equivalently, a non-vanishing section of $\tau^{*} E$. As in the proof of Theorem 5.2 we let $\hat{\omega}=-\hat{c}_{1}\left(L_{D}\right)$. Let $e^{-\psi}$ be the induced metric on $L_{D}^{-1} \otimes \tau^{*} L$. Then, cf. the proof of Theorem 5.2, $1=\left|\sigma^{\prime}\right|^{2}=\left|\sigma^{\prime}\right|_{\circ} e^{-\psi}$ so that $\left|\sigma^{\prime}\right|_{\circ}^{2}=e^{\psi}$ and hence

$$
d d^{c} \log \left|\sigma^{\prime}\right|_{\circ}^{2}=d d^{c} \psi=\hat{\omega}+\tau^{*} \hat{\omega}_{L} .
$$

It follows from (9.7) and (9.12) that ${ }^{10}$

$$
M^{L, \sigma} \wedge \mu=\tau_{*}\left([D] \wedge \frac{1}{1-\hat{\omega}-\tau^{*} \hat{\omega}_{L}} \wedge \alpha\right) .
$$

We have, cf. (5.9),

$$
\begin{aligned}
\sum_{j \geq 0}\left(\frac{1}{1-\hat{\omega}_{L}}\right)^{j} \wedge M_{j}^{\sigma} \wedge \mu & =\sum_{j \geq 0}\left(\frac{1}{1-\hat{\omega}_{L}}\right)^{j+1} \wedge \tau_{*}\left([D] \wedge \hat{\omega}^{j} \wedge \alpha\right) \\
& =\frac{1}{1-\hat{\omega}_{L}} \wedge \tau_{*}\left([D] \wedge \sum_{j \geq 0}\left(\frac{\hat{\omega}}{1-\tau^{*} \hat{\omega}_{L}}\right)^{j} \wedge \alpha\right) \\
& =\frac{1}{1-\hat{\omega}_{L}} \wedge \tau_{*}\left([D] \wedge \frac{1-\tau^{*} \hat{\omega}_{L}}{1-\tau^{*} \hat{\omega}_{L}-\hat{\omega}} \wedge \alpha\right)=M^{L, \sigma} \wedge \mu
\end{aligned}
$$

Thus (9.10) follows, and (9.11) is proved in a similar way.

Corollary 9.9 If $\mu \in \mathcal{B}_{k}(X)$ and $\omega_{L}=c_{1}(L)$ we have

$$
V(\mathcal{J}, L, \mu)=\sum_{j \geq 0}\left(\frac{1}{1-\omega_{L}}\right)^{j} \wedge S_{j}(\mathcal{J}, \mu), S(\mathcal{J}, \mu)=\sum_{j \geq 0}\left(\frac{1}{1+\omega_{L}}\right)^{j} \wedge V_{j}(\mathcal{J}, L, \mu)
$$

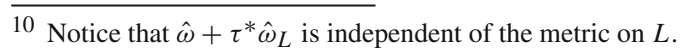


Remark 9.10 Suppose that $\mu=\mathbf{1}_{X}$. Fix $k \geq 0$ and consider the decomposition, cf. (3.10), $M_{k}^{L, \sigma}=M_{k, f i x}^{L, \sigma}+M_{k, \text { mov }}^{L, \sigma}$ of the component $M_{k}^{L, \sigma}$ of codimension $k$. Since $M_{k}^{L, \sigma}$ is obtained as a mean value of $v_{k}^{a \cdot \sigma}$, which are cycles of pure codimension $k$, it is clear that any irreducible $k$-cycle $V$ of $X$ that occurs in all generic SV-cycles must appear in $M_{k, f i x}^{L, \sigma}$. In the literature, such a cycle $V$ is called a fixed component; any other component in a generic SV-cycle is called a moving component. Since the Zariski support of the irreducible components of $M_{k, \text { mov }}^{L, \sigma}$ have codimension strictly smaller than $k$, they must be mean values of moving components of $v_{k}^{a \cdot \sigma}$. It follows from (9.14) that the fixed components of $V_{k}(\mathcal{J}, L, X)$ and $S_{k}(\mathcal{J}, X)$ are the same, cf. (4.10).

Example 9.11 Assume that $X=\mathbb{P}_{\left[z_{0}: \cdots: z_{d}: w_{1}: \cdots: w_{\kappa}\right]}^{d+\kappa}$, let $L:=\mathcal{O}(1)$, and let $i: Z \rightarrow X$ be the linear subspace $\left\{w_{1}=\cdots=w_{\kappa}=0\right\}$. If $\mathcal{J}=\mathcal{J}\left(w_{1}, \ldots, w_{\kappa}\right)$, then $Z=$ $Z_{\mathcal{J}}$ is a smooth (hence regular) embedding defined by the section $w$ of $E=\oplus_{1}^{\kappa} L$. Thus $\left.E\right|_{Z} \simeq N_{\mathcal{J}} X$, cf. (7.5). We want to compute the $\mathcal{B}$-Gysin mapping (1.8), or more precisely (1.9), in this case. If we equip $L$ with the Fubini-Study metric $\hat{\omega}_{L}=$ $d d^{c} \log \left(|z|^{2}+|w|^{2}\right)_{\circ}$, then $\hat{c}(L)=1+\hat{\omega}_{L}$, and therefore

$$
\hat{c}\left(N_{\mathcal{J}} X\right)=\left(1+\hat{\omega}_{L}\right)^{\kappa}
$$

Let $\mu \in \mathcal{G Z}_{k}(X)$. By (9.11)

$$
\begin{aligned}
\left(\hat{c}\left(N_{\mathcal{J}} X\right) \wedge M^{w} \wedge \mu\right)_{k-\kappa} & =\left(\left(1+\hat{\omega}_{L}\right)^{\kappa} \wedge M^{w} \wedge \mu\right)_{k-\kappa} \\
& =\left(\sum_{j \geq 0}\left(1+\hat{\omega}_{L}\right)^{\kappa-j} \wedge M_{j}^{L, w} \wedge \mu\right)_{k-\kappa}=\sum_{j \geq 0} \hat{\omega}_{L}^{\kappa-j} \wedge M_{j}^{L, w} \wedge \mu .
\end{aligned}
$$

By (9.16) one can thus reduce the computation of (1.9) to find $M_{j}^{L, w} \wedge \mu$, which in turn can be obtained as mean values of generic SV-cycles.

From Theorem 9.8 and Lemma 6.2 we have

Proposition 9.12 For each $x \in X$, $\operatorname{mult}_{x}\left(M_{k}^{L, \sigma} \wedge \mu\right)=\operatorname{mult}_{x}\left(M_{k}^{\sigma} \wedge \mu\right)$.

For $\mu \in \mathcal{G Z}_{d}(X)$ we let

$$
\operatorname{deg}_{L} \mu:=\int_{X} \hat{\omega}_{L}^{d} \wedge \mu
$$

where $\hat{\omega}_{L}$ is any representative of $c_{1}(L)$; by Stokes' theorem it is well-defined. Moreover, in view of (4.2) it only depends on the image of $\mu$ in $\mathcal{B}_{d}(X)$ and so $\operatorname{deg}_{L}$ is well-defined on $\mathcal{B}(X)$. If $\mu$ is a cycle, then $\operatorname{deg}_{L} \mu$ is the usual degree of $\mu$ with respect to $L$. The degree is indeed the mass with respect to $L$ of $\mu$, and we have the following mass formula: 
Proposition 9.13 If $\mathcal{J} \rightarrow X$ is generated by the tuple $\sigma=\left(\sigma_{0}, \ldots, \sigma_{m}\right)$ of sections of $L \rightarrow X$ and $\mu \in \mathcal{G Z}_{d}(X)$, then

$\operatorname{deg}_{L} \mu=\operatorname{deg}_{L}\left(M_{0}^{L, \sigma} \wedge \mu\right)+\cdots+\operatorname{deg}_{L}\left(M_{d}^{L, \sigma} \wedge \mu\right)+\operatorname{deg}_{L}\left(\mathbf{1}_{X \backslash Z}\left(d d^{c} \log |\sigma|_{\circ}^{2}\right)^{d} \wedge \mu\right)$.

If $m+1 \leq d$, then the last term on the right hand side vanishes.

Proof We can assume that $\mu=\tau_{*} \alpha$ where $\tau: W \rightarrow X$ and $W$ is connected. If $\mathcal{J}$ vanishes identically on $\mu$, then both sides of (9.17) are equal to deg $L \mu$. Otherwise we may assume that $\tau^{*} \sigma=\sigma^{0} \sigma^{\prime}$ where $\sigma^{0}$ is a section of the line bundle $L_{D}$ defining the divisor $D$ on $W$ and $\sigma^{\prime}$ is a non-vanishing section of $\tau^{*} E \otimes L_{D}^{-1}$, where $E=\oplus_{0}^{m} L$. Notice that in view of (2.1),

$$
\operatorname{deg}_{L} \mu=\int_{W} \tau^{*} \hat{\omega}_{L}^{d} \wedge \alpha .
$$

Let $\hat{\omega}=-\hat{c}_{1}\left(L_{D}\right)$. By (9.12), $\omega_{\sigma}:=d d^{c} \log \left|\sigma^{\prime}\right|_{\circ}^{2}=\hat{\omega}+\tau^{*} \hat{\omega}_{L}$. From (5.5) thus

$$
\tau^{*} \hat{\omega}_{L}=[D]+\omega_{\sigma}+d d^{c} v
$$

where $v=-\log \left|\tau^{*} \sigma\right|$, which is a a global integrable form on $W$. By repeated use of Stokes' theorem we get

$$
\begin{aligned}
\int_{W} \tau^{*} \hat{\omega}_{L}^{d} \wedge \alpha= & \int_{W} \tau^{*} \hat{\omega}_{L}^{d-1} \wedge[D] \wedge \alpha+\int_{W} \tau^{*} \hat{\omega}_{L}^{d-1} \wedge \omega_{\sigma} \wedge \alpha \\
= & \int_{W} \tau^{*} \hat{\omega}_{L}^{d-1} \wedge[D] \wedge \alpha+\int_{W} \tau^{*} \hat{\omega}_{L}^{d-2} \wedge[D] \wedge \omega_{\sigma} \wedge \alpha+\int_{W} \tau^{*} \hat{\omega}_{L}^{d-2} \wedge \omega_{\sigma}^{2} \wedge \alpha \\
= & \int_{W} \tau^{*} \hat{\omega}_{L}^{d-1} \wedge[D] \wedge \alpha+\int_{W} \tau^{*} \hat{\omega}_{L}^{d-2} \wedge \omega_{\sigma} \wedge[D] \wedge \alpha+\cdots+\int_{W} \omega_{\sigma}^{d-1} \wedge[D] \wedge \alpha \\
& +\int_{W} \omega_{\sigma}^{d} \wedge \alpha .
\end{aligned}
$$

Now (9.17) follows from the proof of Proposition 9.3, cf. (9.6) and (9.7), since

$$
\mathbf{1}_{X \backslash Z}\left(d d^{c} \log |\sigma|_{\circ}^{2}\right)^{d} \wedge \mu=\tau_{*}\left(\omega_{\sigma}^{d} \wedge \alpha\right) .
$$

The last statement follows since $\left(d d^{c} \log \left|\sigma^{\prime}\right|_{\circ}^{2}\right)^{m+1}=0$.

If $u$ is $\hat{\omega}_{L}$-plurisubharmonic with analytic singularities, then one can define $\left(d d^{c} u\right)^{k}$ for any $k$ and an analogous mass formula was proved in [6], see [6, Theorem 1.2].

Remark 9.14 Assume that $\sigma$ and $\mu=\tau_{*} \alpha$ are as in the previous proof. If $g$ is a section of $L \otimes \mathcal{J}$, then $\tau^{*} g=\sigma^{0} g^{\prime}$ where $g^{\prime}$ is a section of $L_{D}^{-1} \otimes \tau^{*} L$. Let $h_{1}, \ldots, h_{d}$ be a $\mathrm{SV}$-sequence on $\mu$ and $v^{h} \wedge \mu$ be the associated SV-cycle. If $h$ is sufficiently generic, then with essentially the same proof we get

$\operatorname{deg}_{L} \mu=\operatorname{deg}_{L}\left(v_{0}^{h} \wedge \mu\right)+\cdots+\operatorname{deg}_{L}\left(v_{d}^{h} \wedge \mu\right)+\operatorname{deg}_{L}\left(\mathbf{1}_{X \backslash Z}\left[\operatorname{div} h_{d}\right] \wedge \cdots \wedge\left[\operatorname{div} h_{1}\right] \wedge \mu\right)$. 
Finally let us consider the special case when $\mu$ is an ordinary cycle. With no loss of generality we can assume that $\mu=\mathbf{1}_{X}$. Let $h=\left(h_{1}, h_{2}, \ldots, h_{n}\right)$ be a sequence of sections of $L \otimes \mathcal{J}$. One can check that $h$ is an SV-sequence on $X$ if and only if

$$
\operatorname{codim}\left((X \backslash Z) \cap\left\{h_{1}=\cdots=h_{k}=0\right\}\right)=k \text { or } \infty, \quad k=1, \ldots, n ;
$$

this is the condition in [20]. The SV-algorithm in [20] is precisely the same as used above and the resulting SV-cycle therefore is, in our notation, cf. (9.3),

$$
v^{h}=\mathbf{1}_{Z}+\mathbf{1}_{Z}\left[\operatorname{div} h_{1}\right]+\cdots+\mathbf{1}_{Z}\left[\operatorname{div} h_{n}\right] \wedge \cdots \wedge\left[\operatorname{div} h_{1}\right] .
$$

Let us now assume that $X$ is irreducible. If $\mathcal{J}$ vanishes identically, then $v^{h}=\mathbf{1}_{X}$ for any $\mathrm{SV}$-sequence, and we define $v(\mathcal{J}, L, X)=v_{0}(\mathcal{J}, L, X)=1$. Otherwise, let us assume that $\tau: X^{\prime} \rightarrow X$ is a modification such that $\tau^{*} \mathcal{J}$ is principal, and let $D$ and $L_{D}$ be as before. In particular, let $\sigma^{0}$ be a section of $L_{D}$ that defines the divisor $D$. Then $\tau^{*} h_{k}=\sigma^{0} h_{k}^{\prime}$ where $h_{k}^{\prime}$ are sections of $L_{D}^{-1} \otimes \tau^{*} L$. As in the proof of Proposition 9.3, cf. [5, Eq. (6.3)], we then have

$$
v_{k}^{h}=\tau_{*}\left([D] \wedge\left[\operatorname{div} h_{k-1}^{\prime}\right] \wedge \cdots \wedge\left[\operatorname{div} h_{1}^{\prime}\right]\right),
$$

where the case $k=1$ shall be interpreted as $\tau_{*}[D]$. Choosing the sequence $h_{j}$ even more generic if necessary, we can in addition assume that all the intersections

$$
\left[\operatorname{div} h_{k-1}^{\prime}\right] \wedge \cdots \wedge\left[\operatorname{div} h_{1}^{\prime}\right] \wedge[D]
$$

are proper. As before, let $\omega_{L}:=c_{1}(L)$ and $\omega=-c_{1}\left(L_{D}\right)$. Then the first Chern class of $L_{D}^{-1} \otimes \tau^{*} L$ is $\omega+\tau^{*} \omega_{L}$. By definition, cf. Section 2, therefore (9.20) is a representative of the Chow class $\left(\omega+\tau^{*} \omega_{L}\right)^{k-1} \cap[D]$. We conclude that a generic $\mathrm{SV}$-sequence defines the Chow class

$$
v(\mathcal{J}, L, X):=\sum_{k \geq 1} \tau_{*}\left(\left(\omega+\tau^{*} \omega_{L}\right)^{k-1} \cap[D]\right)=\tau_{*}\left(\frac{1}{1-\omega-\tau^{*} \omega_{L}} \cap[D]\right) .
$$

It follows that this class only depends on $L$ and $\mathcal{J}$ but not on the choice of modification of $X$. If $X$ is not irreducible and consists of the irreducible components $X^{1}, X^{2}, \ldots$, then we define $v(\mathcal{J}, L, X)=v\left(\mathcal{J}, L, X^{1}\right)+v\left(\mathcal{J}, L, X^{2}\right)+\cdots$. The formulas

$$
v(\mathcal{J}, L, X)=\sum_{j \geq 0}\left(\frac{1}{1-\omega_{L}}\right)^{j} \cap s_{j}(\mathcal{J}, X), \quad s(\mathcal{J}, X)=\sum_{j \geq 0}\left(\frac{1}{1+\omega_{L}}\right)^{j} \cap v_{j}(\mathcal{J}, L, X),
$$

are due to van Gastel, [14, Corollary 3.7], and can be obtained by mimicking the proof of Theorem 9.8 above. 


\section{Comparison of $\mathcal{A}(X)$ and $\mathcal{B}(X)$}

In this section we assume that $X$ is compact and projective. In particular, each line bundle over $X$ has a nontrivial meromorphic section. Let $\widehat{H}^{k, k}(X)$ be the equivalence classes of $d$-closed $(k, k)$-currents $\mu$ on $X$ of order zero such that $\mu \sim 0$ if there is a current $\gamma$ of order zero such that $\mu=d \gamma$. Notice that if $i: X \rightarrow Y$ is an embedding into a smooth manifold $Y$ of dimension $N$, then there is a natural mapping $i_{*}: \widehat{H}^{n-k, n-k}(X) \rightarrow H^{N-k, N-k}(Y, \mathbb{C})$ induced by the push-forward of currents. If $X$ is smooth and $X=Y$, then this map gives an isomorphism $\widehat{H}^{n-k, n-k}(X) \simeq$ $H^{n-k, n-k}(X, \mathbb{C})$; the surjectivity is clear and the injectivity follows since a closed current of order zero locally has a potential of order zero.

Example 10.1 Assume that $h$ is a meromorphic section of a Hermitian line bundle $L \rightarrow$ $X$ such that div $h$ intersects $\mu \in \mathcal{G Z}_{k}(X)$ properly. It follows from Proposition 8.1 that [div $h] \wedge \mu$ and $\hat{c}_{1}(L) \wedge \mu$ coincide in $\widehat{H}^{1,1}(X)$.

Let $E \rightarrow X$ be a Hermitian vector bundle. Since $\hat{c}_{k}(E)$ is smooth and closed on $X$, $\mu \mapsto \hat{c}_{k}(E) \wedge \mu$ is a well-defined mapping on $\widehat{H}(X)$. Another choice of metric gives rise to a smooth form that is $\hat{c}_{k}(E)+d d^{c} \psi$ for a suitable smooth form $\psi$ (if $k \geq 1$ ). Thus we get a mapping $\mu \mapsto c_{k}(E) \wedge \mu$ on $\widehat{H}(X)$. Let $0 \rightarrow S \rightarrow E \rightarrow Q \rightarrow 0$ be a short exact sequence of Hermitian vector bundles on $X$. Then, cf. Sect. 4 , there is a smooth $\gamma$ on $X$ such that $d d^{c} \gamma=\hat{c}(E)-\hat{c}(S) \wedge \hat{c}(Q)$. Thus

$$
(c(E)-c(S) \wedge c(Q)) \wedge \mu=0, \quad \mu \in \widehat{H}(X) .
$$

In view of (4.2) there is a natural mapping

$$
B_{X}: \mathcal{B}_{k}(X) \rightarrow \widehat{H}^{n-k, n-k}(X), \quad k=0,1,2 \ldots
$$

If $f: X^{\prime} \rightarrow X$ is a proper map, then

$$
B_{X} f_{*} \mu=f_{*} B_{X^{\prime}} \mu \text {. }
$$

If $E \rightarrow X$ is a vector bundle, then

$$
B_{X}(c(E) \wedge \mu)=c(E) \wedge B_{X} \mu, \quad \mu \in \mathcal{B}(X) .
$$

In view of (2.17) there is a mapping

$$
A_{X}: \mathcal{A}_{k}(X) \rightarrow \widehat{H}^{n-k, n-k}(X), \quad k=0,1,2 \ldots,
$$

taking a representative $\hat{\mu}$ of $\mu$ to the cohomology class determined by its Lelong current. Clearly

$$
A_{X} \mu=B_{X} \mu, \quad \mu \in \mathcal{Z}(X) ;
$$


as a consequence, the image of $A_{X}$ is contained in the image of $B_{X}$. If $f: X^{\prime} \rightarrow X$ is proper as above we have from (2.13) that

$$
A_{X} f_{*} \mu=f_{*} A_{X^{\prime}} \mu, \quad \mu \in \mathcal{A}(X) .
$$

We will use the equalities, see [12, Theorem 3.2],

$$
c(E) \cap f_{*} \mu=f_{*}\left(c\left(f^{*} E\right) \cap \mu\right),
$$

in $\mathcal{A}(X)$ if $E \rightarrow X$ is a vector bundle, and

$$
c(E) \cap \mu=c(L) \cap(c(Q) \cap \mu)
$$

in $\mathcal{A}(X)$ if $0 \rightarrow L \rightarrow E \rightarrow Q \rightarrow 0$ is exact. In analogy with (10.3) we have:

$$
A_{X}(c(E) \cap \mu)=c(E) \wedge A_{X} \mu
$$

Proof of Eq. (10.8) First assume that $E=L$ has rank 1; it is then sufficient to show (10.8) for $c_{1}(L)$. By $\mathbb{Z}$-linearity it is enough to look at each irreducible component of $\mu$ separately and so we may assume that $\mu$ is represented in $\mathcal{A}(X)$ by an irreducible subvariety $V \hookrightarrow X$. Let $h$ be a meromorphic section of $L$ that is nontrivial on $V$. Then $c_{1}(L) \cap \mu$ is represented in $\mathcal{A}(X)$ by the cycle $[\operatorname{div} h] \wedge[V]$ and so

$$
A_{X}\left(c_{1}(L) \cap \mu\right)=[\operatorname{div} h] \wedge[V]=c_{1}(L) \wedge[V]=c_{1}(L) \wedge A_{X} \mu
$$

in $\widehat{H}(X)$, where the second equality follows from Example 10.1 applied to [V].

Next, assume that (10.8) holds for vector bundles of rank $\leq r$ and consider $E$ of rank $r+1$. Let $p: X^{\prime} \rightarrow X$, where $X^{\prime}=\mathbb{P}(E)$, and let $L=\mathcal{O}(-1)$ be the tautological line subbundle so that we have a short exact sequence $0 \rightarrow L \rightarrow p^{*} E \rightarrow Q \rightarrow 0$ over $X^{\prime}$. Take $\mu^{\prime}$ in $\mathcal{A}\left(X^{\prime}\right)$ such that $p_{*} \mu^{\prime}=\mu$. By (10.6) and (10.7),

$$
c(E) \cap \mu=c(E) \cap p_{*} \mu^{\prime}=p_{*}\left(c\left(p^{*} E\right) \cap \mu^{\prime}\right)=p_{*}\left(c(L) \cap\left(c(Q) \cap \mu^{\prime}\right)\right) .
$$

By (2.1), (2.8), (10.1), (10.5), (10.9), and the induction hypothesis

$$
\begin{aligned}
A_{X}(c(E) \cap \mu) & =A_{X} p_{*}\left(c(L) \cap\left(c(Q) \cap \mu^{\prime}\right)\right) \\
& =p_{*} A_{X^{\prime}}\left(c(L) \cap\left(c(Q) \cap \mu^{\prime}\right)\right)=p_{*}\left(c(L) \wedge A_{X^{\prime}}\left(c(Q) \cap \mu^{\prime}\right)\right) \\
& =p_{*}\left(c(L) \wedge c(Q) \wedge A_{X^{\prime}} \mu^{\prime}\right)=p_{*}\left(c\left(p^{*} E\right) \wedge A_{X^{\prime}} \mu^{\prime}\right) \\
& =c(E) \wedge p_{*}\left(A_{X^{\prime}} \mu^{\prime}\right)=c(E) \wedge A_{X} \mu .
\end{aligned}
$$

Proof of Proposition 1.6 We have already noticed, (10.4), that the image of $A_{X}$ is contained in the image of $B_{X}$. For the converse inclusion consider $\mu=\tau_{*} \alpha$ in $\mathcal{G Z}_{k}(X)$, $\tau: W \rightarrow X$. By (10.5), (10.8), (10.4), (10.3), and (10.2) we have

$$
A_{X} \tau_{*}\left(\alpha \cap \mathbf{1}_{W}\right)=\tau_{*} A_{W}\left(\alpha \cap \mathbf{1}_{W}\right)=\tau_{*}\left(\alpha \wedge A_{W} \mathbf{1}_{W}\right)
$$




$$
=\tau_{*}\left(\alpha \wedge B_{W} \mathbf{1}_{W}\right)=\tau_{*} B_{W}\left(\alpha \wedge \mathbf{1}_{W}\right)=B_{X} \tau_{*}\left(\alpha \wedge \mathbf{1}_{W}\right)=B_{X} \mu
$$

and thus $B_{X} \mu$ is in the image of $A_{X}$.

Proof of Proposition 1.7 Let $N=N_{\mathcal{J}} X$. We may assume that $\mu$ is an irreducible subvariety $i: V \rightarrow X$. If $\mathcal{J}$ vanishes identically on $V$, then $\mu$ is mapped to $\mu$ under both the Gysin and the $\mathcal{B}$-Gysin mapping. Thus we can assume that we have a modification $\pi: V^{\prime} \rightarrow V$ such that $\pi^{*} i^{*} \mathcal{J}$ is principal on $V^{\prime}$. Let $D$ be the exceptional divisor and $L=L_{D}$ the associated line bundle. Using (10.4), (10.5), and (10.8), recalling that $s(\mathcal{J}, V)=s\left(i^{*} \mathcal{J}, V\right)$, see the introduction and Remark 5.1, we have

$$
\begin{aligned}
A_{Z}(c(N) \cap s(\mathcal{J}, V)) & =A_{Z}\left(c(N) \cap s\left(i^{*} \mathcal{J}, V\right)\right)=A_{Z}\left(c(N) \cap \pi_{*}\left(\frac{1}{c(L)} \cap[D]\right)\right) \\
& =c(N) \wedge A_{Z} \pi_{*}\left(\frac{1}{c(L)} \cap[D]\right)=c(N) \wedge \pi_{*} A_{|D|}\left(\frac{1}{c(L)} \cap[D]\right) \\
& =c(N) \wedge \pi_{*}\left(\frac{1}{c(L)} \wedge A_{|D|}[D]\right)=c(N) \wedge \pi_{*}\left(\frac{1}{c(L)} \wedge B_{|D|}[D]\right) .
\end{aligned}
$$

By an analogous computation backwards with $B_{|D|}$ rather than $A_{|D|}$, using (10.3) and (10.2), we find that the right hand side of (10.10) is equal to $B_{Z}(c(N) \wedge S(\mathcal{J}, V))$.

Notice in particular that $A_{Z} s(\mathcal{J}, X)=B_{Z} S(\mathcal{J}, X)$. Summing up we have seen that the $\mathcal{A}$ - and $\mathcal{B}$-objects coincide on cohomology level. However, there are no nontrivial mappings $T_{X}: \mathcal{A}_{k}(X) \rightarrow \mathcal{B}_{k}(X)$ such that

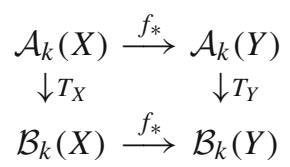

commutes for each proper mapping mapping $f: X \rightarrow Y$. In fact, let $X$ be a onepoint set $\{0\}$, and let $Y$ be a manifold with two distinct points $p, q$ that are rationally equivalent. Take $f$ so that $f(0)=p$. If $T_{X}$ is nonzero, then $f_{*} T_{X}[0]$ has support at $p$ and is nonzero. If (10.11) commutes, then $T_{Y}[q]=T_{Y}[p]$ must be a nonzero point mass at $p$. Changing the roles of $p$ and $q$ we get a contradiction since $[p] \neq[q]$ in $\mathcal{B}(Y)$.

Neither there are non-trivial mappings $T_{X}: \mathcal{B}_{k}(X) \rightarrow \mathcal{A}_{k}(X)$ such that

$$
\begin{array}{ccc}
\mathcal{B}_{k}(X) & \stackrel{B_{X}}{\longrightarrow} \widehat{H}^{k, k}(X) \\
\downarrow T_{X} & & \downarrow I d \\
\mathcal{A}_{k}(X) & \stackrel{A_{X}}{\longrightarrow} & \widehat{H}^{k, k}(X)
\end{array}
$$

commutes and $T_{X}\left(c_{1}(L) \wedge \mu\right)=c_{1}(L) \cap T_{X} \mu$ for each line bundle $L$. Just take $X$ that has a nontrivial line bundle with flat metric and a meromorphic non-trivial section, as in the following example. 
Example 10.2 Let $X$ be a complex 1-dimensional torus. It is well-known that two different points $p_{1}$ and $p_{2}$ are not rationally equivalent, i.e., there is no meromorphic function whose divisor is $[D]:=\left[p_{1}\right]-\left[p_{2}\right]$. But the cohomology class determined by $[D]$ is zero. Let $L$ be the line bundle $\mathcal{O}(D)$ equipped with some Hermitian metric. By the Poincaré-Lelong formula, $\hat{c}_{1}(L)$ is a representative of the cohomology class of $[D]$ and is thus $d$-exact. Hence, since $\hat{c}_{1}(L)$ is smooth, the $d d^{c}$-lemma shows that there is a smooth global function $\phi$ such that $d d^{c} \phi=\hat{c}_{1}(L)$. If we modify the metric on $L$ by $\exp (-\phi)$ we have $\hat{c}_{1}(L)=0$.

Acknowledgements Open access funding provided by University of Gothenburg.

Open Access This article is licensed under a Creative Commons Attribution 4.0 International License, which permits use, sharing, adaptation, distribution and reproduction in any medium or format, as long as you give appropriate credit to the original author(s) and the source, provide a link to the Creative Commons licence, and indicate if changes were made. The images or other third party material in this article are included in the article's Creative Commons licence, unless indicated otherwise in a credit line to the material. If material is not included in the article's Creative Commons licence and your intended use is not permitted by statutory regulation or exceeds the permitted use, you will need to obtain permission directly from the copyright holder. To view a copy of this licence, visit http://creativecommons.org/licenses/by/4.0/.

\section{References}

1. Achilles, R., Manaresi, M.: Multiplicities of a bigraded ring and intersection theory. Math. Ann. 309, 573-591 (1997)

2. Achilles, R., Rams, S.: Intersection numbers, Segre numbers and generalized Samuel multiplicities. Arch. Math. (Basel) 77, 391-398 (2001)

3. Andersson, M.: Residue currents of holomorphic sections and Lelong currents. Arkiv för Matematik 43, 201-219 (2005)

4. Andersson, M., Samuelsson Kalm, H.: A note on smooth forms on analytic spaces. arXiv:2003.01959

5. Andersson, M., Samuelsson Kalm, H., Wulcan, E., Yger, A.: Segre numbers, a generalized King formula, and local intersections. J. Reine Angew. Math 728, 105-136 (2017)

6. Andersson, M., Błocki, Z., Wulcan, E.: On a Monge-Ampère operator for plurisubharmonic functions with analytic singularities. Indiana Univ. Math. J. 68(4), 1217-1231 (2019)

7. Andersson, M., Eriksson, D., Samuelsson Kalm, H., Wulcan, E., Yger, A.: Nonproper intersection products and generalized cycles. arXiv:1908.11759

8. Bost, J.-B., Gillet, H., Soulé, C.: Heights of projective varieties and positive Green forms. J. A. Math. Soc. 7, 903-1027 (1994)

9. Bott, R., Chern, S.S.: Hermitian vector bundles and the equidistribution of the zeroes of their holomorphic sections. Acta Math 114, 71-112 (1965)

10. Chirka, E.M.: Complex Analytic Sets. Kluwer, Dordretch (1989)

11. Demailly, J. P. Complex analytic and differential geometry. On-line book available at https://wwwfourier.ujf-grenoble.fr/ demailly/manuscripts/agbook.pdf

12. Fulton, W.: Intersection Theory, 2nd edn. Springer-Verlag, Berlin-Heidelberg (1998)

13. Gaffney, T., Gassler, R.: Segre numbers and hypersurface singularities. J. Algebraic Geom. 8, 695-736 (1999)

14. van Gastel, L.J.: Excess intersections and a correspondence principle. Invent. Math. 103, 197-222 (1991)

15. Gillet, H., Soulé, C.: Arithmetic intersection theory. Inst. Hautes Études Sci. Publ. Math. 72, 93-174 (1990)

16. Griffiths, P., King, J.R.: Nevanlinna theory and holomorphic mappings between algebraic varieties. Acta Math. 130, 145-220 (1973)

17. King, J.R.: A residue formula for complex subvarieties. IN: Proc. Carolina conf. on holomorphic mappings and minimal surfaces, Univ. of North Carolina, Chapel Hill, pp. 43-56 (1970) 
18. Lazarsfeld, R.: Positivity in Algebraic Geometry: Classical Setting: Line Bundles and Linear Series. Springer-Verlag, Berlin (2004)

19. Mourougane, C.: Computations of Bott-Chern classes on $\mathbb{P}(E)$. Duke Math. J. 124, 389-420 (2004)

20. Stückrad, J., Vogel, W.: An algebraic approach to the intersection theory. Queen's Papers Pure Appl. Math. 61, 1-32 (1982)

21. Tworzewski, P.: Intersection theory in complex analytic geometry. Ann. Polon. Math. 62, 177-191 (1995)

Publisher's Note Springer Nature remains neutral with regard to jurisdictional claims in published maps and institutional affiliations. 